\title{
A putative long noncoding RNA-encoded micropeptide maintains cellular homeostasis in
} pancreatic $\beta$-cells

Mark $\mathrm{Li}^{1,2,3 \#}$, Fan Shao, ${ }^{4,6 \#}$, Qingwen Qian ${ }^{1,2,3}$, Wenjie $\mathrm{Yu}^{3,7}$, Zeyuan Zhang ${ }^{1,2,3}$, Biyi Chen ${ }^{2,7}$, Dan Su ${ }^{1,4}$, Yuwei Guo ${ }^{4}$, An-Vi Phan ${ }^{4}$, Long-sheng Song ${ }^{2,7}$, Samuel B. Stephens ${ }^{2,3,7}$, Julien Sebag ${ }^{2,3,8}$, Yumi Imai $^{2,3,7}$, Ling Yang ${ }^{1,2,3 *}$, Huojun $\mathrm{CaO}^{2,4,5,6 *}$

${ }^{1}$ Department of Anatomy and Cell Biology; ${ }^{2}$ Fraternal Order of Eagles Diabetes Research Center; ${ }^{3}$ Pappajohn Biomedical Institute; ${ }^{4}$ Iowa Institute for Oral Health Research, ${ }^{5}$ Division of Biostatistics and Computational Biology, ${ }^{6}$ Department of Endodontics; ${ }^{7}$ Department of Internal Medicine; ${ }^{8}$ Department of Molecular Physiology and Biophysics, University of lowa Carver College of Medicine $(1,2,3,7,8)$ and University of lowa College of Dentistry (4,5,6), lowa City, IA 52242

\# Co-first author

* Co-responding author

${ }^{*}$ Address all correspondence to:

Huojun Cao, Ph.D.

lowa Institute for Oral Health Research

Division of Biostatistics and Computational Biology

Department of Endodontics

University of lowa College of Dentistry, lowa City, 52242

Email: huojun-cao@uiowa.edu

Or *Address all correspondence to:

Ling Yang, Ph.D.

Department of Anatomy and Cell Biology

Fraternal Order of Eagles Diabetes Research Center

Pappajohn Biomedical Institute

University of lowa Carver College of Medicine, lowa City, IA 52242

Email: ling-yang@uiowa.edu 


\section{ABSTRACT}

2 Micropeptides (microproteins) encoded by transcripts previously annotated as long noncoding RNA 3 (IncRNAs) are emerging as important mediators of fundamental biological processes in health and disease.

4 Here we applied two computational tools to identify putative micropeptides encoded by IncRNAs that are expressed in the human pancreas. We experimentally verified one such micropeptide encoded by a $\beta$-celland neural cell-enriched IncRNA TUNAR (also known as TUNA, HI-LNC78 or LINC00617). We named this highly conserved 48-amino-acid micropeptide Beta cell- and Neural cell-regulin (BNLN). BNLN contains a single-pass transmembrane domain and localized at the endoplasmic reticulum in pancreatic $\beta$-cells.

9 Overexpression of BNLN lowered ER calcium levels, increased cytosolic calcium levels, and maintained

10 ER homeostasis in response to high glucose challenge. To determine the physiological and pathological 11 roles of BNLN, we assessed the BNLN expression in islets from mice fed with a high-fat diet and a regular 12 diet, and found that BNLN is suppressed by diet-induced obesity (DIO). Conversely, overexpression of 13 BNLN elevated glucose-stimulated insulin secretion in INS-1 cells. Lastly, BNLN overexpression enhanced 14 insulin secretion in islets from lean and obese mice as well as from humans. Taken together, our study 15 provides the first evidence that IncRNA-encoded micropeptides play a critical role in pancreatic $\beta$-cell 16 function and provides a foundation for future comprehensive analyses of micropeptide function and 17 pathophysiological impact on diabetes.

\section{Abbreviations:}

20 ATF6: activating transcription factor-6; BNLN: beta cell and neuron regulin; Cre: Cre recombinase; DIO: 21 diet-induced obesity; ER: endoplasmic reticulum; GAPDH: glyceraldehyde-3-phosphate dehydrogenase; GFP: green fluorescent protein; Glc: glucose; HFD: high-fat diet; IncRNA: long noncoding RNA; RD: regular 
INTRODUCTION:

Large-scale projects such as ENCODE and FANTOM have found thousands of long noncoding RNAs

(IncRNAs) in the human genome ${ }^{1-4}$. IncRNAs play an essential role in regulating islet function in health and

diseases including diabetes ${ }^{5}$. It has been demonstrated that IncRNAs are key components of the islet regulatome, with more than 1000 IncRNAs identified in human islets and mouse $\beta$-cells ${ }^{6,7}$. These IncRNAs play critical roles in islet and $\beta$-cell survival, cell development, and physiological function through regulation of the islet transcriptome ${ }^{5-7}$. Of note, a genome-wide association study that examined T2D susceptibility loci demonstrated that dysregulation of IncRNAs is involved in pancreatic pathologies in humans ${ }^{6}$. In addition, dysregulation of IncRNAs has been implicated in diabetes complications, such as diabetic nephropathy and retinopathy ${ }^{5,8}$. Recently, several circulating IncRNAs have been identified as biomarkers of diabetes and diabetic complications ${ }^{9,10}$. However, although much knowledge has accumulated correlating dysregulation of IncRNA with islet pathology, the functional roles and molecular mechanisms of these IncRNAs remain poorly understood.

By definition, IncRNAs do not contain protein-coding open reading frames (ORF), which comprise a start codon, in-frame codons, and a stop codon. To accurately identify bona fide protein-coding ORFs, most ORF-finding computational algorithms have historically used a cutoff of 100 amino acids as the minimum size for detection. This filtering strategy dramatically reduces the likelihood of false-positive detection, but may also result in some genes that contain small open reading frames (sORFs) being mis-annotated as IncRNAs ${ }^{11}$. Indeed, recent studies have revealed that some of IncRNAs do contain sORFs that encode micropeptides (or microprotein, protein less than 100 amino acids in length) ${ }^{12-20}$. There is emerging evidence that micropeptides are important regulators in fundamental biological processes such as embryonic development ${ }^{21}$ and metabolism ${ }^{22,23}$. However, whether and how micropeptides modulate islet biology is largely unknown.

51 In this study, we employed two computational algorithms, PhyloCSF and RNAcode, to comprehensively 
53 novel micropeptides encoding IncRNAs transcripts we identified, we characterized one putative 54 micropeptide encoded by TUNAR (also known as TUNA, HI-LNC78 or LINC00617), which is expressed highly in human $\beta$-cells and neural cells. We then named this highly conserved micropeptide beta cell and neural cell regulin $(B N L N)$. Pancreatic $\beta$-cells synthesize and secrete insulin to govern systemic glucose homeostasis ${ }^{26}$. We found BNLN localizes at the endoplasmic reticulum (ER) in pancreatic $\beta$-cells. Robust and highly functional endoplasmic reticulum (ER) dynamics are required for $\beta$-cells to synthesize insulin to maintain systemic glucose metabolic homeostasis ${ }^{27}$. The ER also plays an important role in coordinating intracellular $\mathrm{Ca}^{2+}$ signaling which regulates insulin secretion ${ }^{28,29}$. In both rodent models and

humans, obesity and diabetes are characterized by ER dysfunction which leads to $\beta$-cell death and impaired islet function ${ }^{30,31}$. We further showed that $B N L N$ is downregulated in islets from obese mice, and gain of function of $B N L N$ increases glucose-induced insulin secretion in islets from obese mice as well as from human donors. These findings provide first evidence of the biological function of pancreatic micropeptides in health and disease.

\section{RESULTS}

\section{An IncRNA, TUNAR, encodes a conserved 48-amino-acid micropeptide in the human pancreas}

Recent studies have discovered that a few IncRNAs harbor small open reading frames (sORFs) that encode sORF-encoded peptides (SEPs) or micropeptides ${ }^{12-20}$. We hypothesized that there are additional transcripts currently annotated as IncRNAs that may encode micropeptides. To prioritize the search for potential micropeptide genes, we used two computational tools, PhyloCSF and RNAcode, to comprehensively evaluate the coding potential of IncRNAs that are expressed in human pancreatic islets $^{24,25}$. We found several IncRNAs that are highly expressed in human pancreatic islets and contained putative sORFs (Fig. 1A). TCL1 Upstream Neural Differentiation-Associated RNA, TUNAR (also known as TUNA, HI-LNC78 or LINC00617) ranked at the top in both the expression level in human islets and the coding potential (Fig. 1A). Of note, its PhyloCSF and RNAcode scores were higher than many recently identified micropeptides (Fig. 1B). In addition to the pancreas, TUNAR is highly expressed in the brain, 
81 associated with reduced insulin content and impaired glucose-stimulated insulin secretion in a human $\beta$ 82 cell line, as well as impaired neural differentiation of mouse embryonic stem cells (mESCs) ${ }^{32,33}$. Genomic 83 annotation indicated that TUNAR spans $\sim 49.2 \mathrm{~kb}$ and has 3 transcript isoforms: NR_132399.1, NR_132398.1 and NR_038861.1 (Fig. 1C). Further analysis of RNA-Seq read splice junctions visualized with sashimi plot revealed that NR_038861.1 is the major isoform in the pancreas (data not shown). Our computational pipeline predicted an SORF at the beginning of the last exon (which is shared among all three transcript isoforms) that is highly conserved among 100 vertebrate genomes assessed by UCSC genome browser PhyloP track (Fig. 1C). Moreover, ribosomal profiling (Ribo-seq) datasets suggest that this region is actively translated (Supplemental Fig. 2A). We then extracted other species' DNA sequences (multiz100way) corresponding to predicted sORF and translated them to protein sequences. The predicted micropeptide sequence was highly conserved (Fig. 1D, Supplemental Fig. 3 and Supplemental Table1) and could be found in 88 species out of the 100 vertebrate species (conserved down to fishes).

To experimentally validate the translation of the predicted SORF within TUNAR, we inserted three reporters (GFP, hRluc and FLAG) CDS without ATG start codon either in-frame or out-of-frame before the stop codon of the full length TUNAR transcript (NR_038861.1; Fig. 2A). As expected, GFP and Renilla luciferase were translated when inserted in-frame (Fig.2B\&C). However, no translation was observed when GFP or hRluc were inserted out-of-frame by deletion of one or two bases between the predicted sORF and GFP/hRluc (Fig. 2B\&C). Consistent with these results, western blot analysis showed a band $\sim 10-k D a$ only when FLAG was inserted in-frame (Fig. 2D). There are 3 potential ATG initiating codons in-frame with the predicted reading frame in TUNAR (Fig. 1D). Multispecies alignment showed that more than half of all the species contain the $1^{\text {st }}$ ATG, all 88 species have the $2^{\text {nd }}$, and 87 species have $3^{\text {rd }}$ ATG of TUNAR (Supplemental

Fig. 3). Therefore, we generated ATG deletion constructs for each of these potential initiating codons and found that removing the $2^{\text {nd }}$ ATG abolished translation of TUNAR (Fig. 2E\&F). Hence, TUNAR contains an sORF from the $2^{\text {nd }}$ ATG start codon to the stop codon that translates to a 48-amino-acid micropeptide. To keep consistency with the previous nomenclature, we named this micropeptide $\underline{B} e t a$ cell- and Neural cellregulin (BNLN). 


\section{BNLN localizes at the endoplasmic reticulum (ER) in pancreatic $\beta$ cells}

BNLN / TUNAR is highly expressed in the human islet (Fig. 2A). In situ hybridization analysis in islets from

human donors showed that BNLN expression is enriched in the insulin producing $\beta$-cells (Fig. 3A), which

is in agreement with the analysis of previous RNA-Seq data ${ }^{32,34}$. Our in silico sequence analysis revealed that BNLN harbors a single-pass transmembrane domain at its C-terminus (Fig. 1D, Supplemental Fig. 2B).

The multispecies alignment showed that this transmembrane domain is extremely conserved and

presented in all 88 species (Fig. 1D, Supplemental Fig. 3). To determine the sub-cellular localization of

BNLN, we generated N-terminal and C-terminal GFP-FLAG tagged BNLN constructs (Fig. 3B), then

performed immunofluorescence analysis in INS-1 cells. As shown in figure 3C, in contrast to the GFP

control, which was dispersed throughout cells, BNLN mainly localized at the endoplasmic reticulum (ER)

in the INS-1 cell. Much weaker signal was detected in lysosomes, mitochondria, peroxisomes, or Golgi in

INS-1 cells (Fig. 3D). To further assess whether BNLN is secreted or expressed extracellularly, we

generated a BNLN construct fused with HiBiT (Fig. 3E). In the Nano-Glo HiBiT detection system, HiBiT-

tagged protein is recognized by the complementing polypeptide $\mathrm{LgBiT}$, the bound complex then reconstitutes a luminescent signal. As shown in figure 3F, BNLN mainly localized intracellularly in INS-1 cells. Taken together, these data show that BNLN is primarily located at the ER of pancreatic $\beta$-cells.

\section{BNLN modulates ER calcium homeostasis and ER function in the $\beta$ cell}

ER calcium homeostasis has a pivotal role in insulin production and secretion in $\beta$-cells ${ }^{26}$. To determine the functional effect of BNLN in the ER, we first assessed the ER $\mathrm{Ca}^{2+}\left(\left[\mathrm{Ca}^{2+}\right]^{\mathrm{ER}}\right)$ level by using an $\mathrm{ER} \mathrm{Ca}^{2+}$ biosensor (ER-GCaMP6-150) in INS-1 cells overexpressing BNLN or the control construct. Overexpression of BNLN lowered $\left[\mathrm{Ca}^{2+}\right]^{\mathrm{ER}}$ level at both the basal $(2.5 \mathrm{mM})$ and high glucose $(17 \mathrm{mM})$ conditions (Fig. 4A\&B), indicating a potential role of BNLN in modulating ER calcium influx. Glucose-stimulated $\beta$-cells display oscillations of the interrelated cytosolic $\mathrm{Ca}^{2+}\left(\left[\mathrm{Ca}^{2+}\right]^{\mathrm{C}}\right)$ and $\left[\mathrm{Ca}^{2+}\right]^{\mathrm{ER}}$. To evaluate the influence of the BNLN on $\left[\mathrm{Ca}^{2+}\right]^{\mathrm{c}}$ homeostasis, we generated FLAG- or V5-tagged $B N L N$ constructs and measured $\left[\mathrm{Ca}^{2+}\right]^{\mathrm{c}}$ by a 
35 glucose conditions (Fig. 4C\&D). We observed this effect with both FLAG- and V5-tagged BNLN. To further assess the effect of BNLN on depolarization-induced calcium dynamics, we measured $\left[\mathrm{Ca}^{2+}\right]^{\mathrm{c}}$ in INS-1 in the absence or presence of $\mathrm{KCl}$. As shown in figure 4E\&F while BNLN overexpression increased high glucose-induced $\left[\mathrm{Ca}^{2+}\right]^{c}$, it did not alter $\mathrm{KCl}$-mediated $\left[\mathrm{Ca}^{2+}\right]^{\mathrm{c}}$ flux, indicating a regulatory role of BNLN independent from depolarization dependent $\left[\mathrm{Ca}^{2+}\right]^{\mathrm{c}}$ flux that is primarily driven by voltage-dependent calcium channel.

Calcium level is tightly regulated to maintain ER chaperone activity and protein folding ${ }^{35}$. We found that BNLN reduced $\left[\mathrm{Ca}^{2+}\right]$ in the ER of INS-1 cells (Fig. 4A\&B). To determine whether the BNLN-mediated lowering $\left[\mathrm{Ca}^{2+}\right]^{E R}$ effect alters ER function, we examined the functional impact of BNLN on ER homeostasis, using an ATF6 luminal domain (ATF6LD) cypridina noctiluca (Cluc) secretion reporter assay in INS-1 cells. In this assay, cells with abundant ER chaperones retain the ATF6LD-Cluc in the ER and secrete minimal luciferase, while cells with limited ER chaperone availability release the chimeric protein, promoting luciferase secretion into the medium ${ }^{36}$. We found that the high glucose challenge $(17 \mathrm{mM})$ led to a decreased ER chaperone availability (increased ATF6LD-cluc secretion) in INS-1 cells, which was ameliorated by BNLN overexpression (decreased ATF6LD-cluc secretion) (Fig. 4G). These data indicate that BNLN-mediated calcium dynamic in the ER did not impair ER function in INS-1 cells. In contrast, BNLN primed the $\beta$-cell ER against the high glucose-induced ER stress. complex ${ }^{12,13,15,20}$. To identify potential BNLN interacting protein partners in pancreatic $\beta$-cells, we performed BNLN-FLAG overexpression. GFP-FLAG overexpression was used as a control construct. One protein enriched in BNLN-FLAG pull down compared to GFP-FLAG pull-down was sarco-endoplasmic reticulum $\mathrm{Ca}^{2+}$-ATPases 3 (SERCA3; Supplemental Table 2), a major calcium transporter in the ER. We further validated this interaction by Co-IP-western blot analysis in HEK 293 cells with BNLN and SERCA3 overexpression (Fig. 4H). To determine the impact of glucose on the interaction of BNLN with SERCA3, 
we further performed a proximity ligation assay (PLA) in INS-1 cells in the presence of low (2.5mM) or high $(17 \mathrm{mM})$ glucose. As shown in figure $4 \mathrm{I}$, a low level of interaction between BNLN and SERCA3 occurred in low glucose conditions $(2.5 \mathrm{mM})$, and this was elevated in the presence of high glucose $(17 \mathrm{mM})$. Taken together, these data indicate an important role of BNLN on modulating calcium dynamics in the pancreatic $\beta$-cells.

\section{BNLN enhances glucose-stimulated insulin secretion}

$\mathrm{Ca}^{2+}$ oscillations in pancreatic $\beta$-cells are critical for their response to changes in glucose level ${ }^{37}$. To

establish the functional role of BNLN in the pancreatic- $\beta$ cell, we first measured glucose-stimulated insulin

secretion (GSIS) in INS-1 cells with BNLN overexpression using adenovirus-mediated gene delivery. As

shown in figure 5A, BNLN overexpression significantly increased GSIS in the INS-1 cells compared to cells

overexpressed with control vector. To verify that this effect was mediated by the translated micropeptide and not the RNA transcript, we transfected INS-1 cells with the $2^{\text {nd }}$ ATG-deletion BNLN construct (which does not produce the micropeptide), and found that this construct did not affect GSIS (Fig. 5B). To further examine the BNLN-mediated insulin secretion, we transduced islets isolated from lean mice with the adenovirus-mediated BNLN construct. As shown in figure 5C, GSIS was significantly enhanced in Islets overexpressing BNLN.

Obesity results in dysregulated GSIS in pancreatic islets. To establish the pathophysiological relevance of

BNLN expression, we next assessed the expression level of BNLN transcripts in islets from mouse islets 82 from lean mice and mice with diet-induced obesity (DIO). BNLN expression was significantly downregulated in islets from obese mice (Fig. 5D). To test whether BNLN could rescue obesity-associated islet dysfunction, we further examined GSIS in lean and obese mouse islets following BNLN overexpression. As shown in figure 5E, overexpression of BNLN significantly improved GSIS in islets from mice with DIO. Finally, to establish the functional relevance of BNLN in humans, we measured GSIS in 
88 increased GSIS in islets from non-diabetic humans with minimal effect on insulin content (Fig. 5F-I). These data strongly implicate that BNLN functions as a small peptide to modulate insulin secretion in $\beta$-cells.

\section{DISSCUSION}

92 Obesity and diabetes are characterized by dysregulation of insulin secretion from pancreatic $\beta$-cells. 93 Although IncRNAs have been implicated in this process ${ }^{5-7}$, thus far there is no evidence of regulatory role 94 of IncRNA-encoded micropeptides in pancreatic $\beta$-cell function. Our study offers the first insight into the 95 impact of micropeptides on $\beta$-cell physiology. We showed that the IncRNA TUNAR (also known as TUNA, HI-LNC78 or LINC00617) encodes BNLN, a 48aa micropeptide. We further demonstrate that BNLN regulates glucose-induced insulin secretion in $\beta$-cells and intact islets in part through modulating ER homeostasis.

LncRNAs regulate key biological processes through regulation of transcriptional activation, heterochromatin formation, interaction with miRNAs and other mechanisms ${ }^{38,39}$. However, recent studies have revealed that some transcripts identified as IncRNAs actually do contain sORFs that encode micropeptide (microprotein) $)^{12-20}$. In this study, we applied two computational programs PhyloCSF and RNAcode to prioritize the search for additional micropeptide encoding IncRNAs expressed in human pancreas. It should be noted that both PhyloCSF and RNAcode depend on the quality of whole genome sequence alignment. False negatives could be resulted from poor or incorrect whole genome sequence alignment. Among the predicted micropeptide encoding IncRNA, we choose one IncRNA, TCL1 Upstream Neural Differentiation-Associated RNA (TUNAR), for further characterization because it ranked at the top of both the expression level in human islets and the coding potential. TUNAR has been shown to be important for ESC pluripotency and neural differentiation ${ }^{40,41}$. Dysregulation of TUNAR has been implicated 11 in the progression of Huntington's Disease ${ }^{42}$ and breast cancer ${ }^{5}$. Notably, a recent study showed that 12 knocking down TUNAR suppresses insulin secretion in a human $\beta$-cell line ${ }^{5}$. Multiple molecular mechanisms have been proposed underlying functions of TUNAR in the context of diverse biology 
15 activate pluripotency genes in a $\beta$-cell line ${ }^{40}$. Our computational and experimental studies identified an sORF encoding BNLN, a 48aa micropeptide within the last exon of TUNAR. Overexpression of this micropeptide regulated GSIS, while deletion of start codon (ATG) abolished its function (Fig. 5B). However, we could not completely rule out a role for TUNAR IncRNA in this process. Therefore, future studies will focus on dissecting the contributions of TUNAR RNA and BNLN micropeptide on the $\beta$-cell transcriptome development and differentiation, and metabolic function.

Accumulating evidence demonstrates that transmembrane micropeptides tightly modulate intracellular and extracellular signaling cascades ${ }^{43}$. We found that BNLN contains a transmembrane domain at the Cterminus. To determine the sub-cellular localization, cellular function, and molecular mechanisms of BNLN in $\beta$-cells, we generated a set of epitope-tagged BNLN constructs. It is possible that epitope tags altered the signal sequences and function of BNLN, however, we did not observe functional differences between the differently tagged BNLN constructs (Fig. 4C). Further studies with specific anti-BNLN antibodies will be necessary to confirm subcellular localization of the endogenous micropeptide.

ER calcium homeostasis is required for insulin production and secretion in $\beta$-cells ${ }^{26}$. $\mathrm{Ca}^{2+}$ serves as a critical co-factor for protein chaperones and foldases needed to support high levels of protein translation and pro-insulin processing ${ }^{35}$. Moreover, the ER of $\beta$-cells contributes to a tight regulation of cytosolic $\mathrm{Ca}^{2+}$ by taking up $\mathrm{Ca}^{2+}$ when the cell is depolarized (cytosolic $\mathrm{Ca}^{2+}$ is elevated), and slowly releasing $\mathrm{Ca}^{2+}$ when the cell is repolarized ${ }^{44}$. In pancreatic islets, the ER primarily takes up cytosolic $\mathrm{Ca}^{2+}$ by two SERCAs: ubiquitously expressed SERCA2b, and SERCA3, which is expressed only in the islet $\beta$-cell ${ }^{45,46}$. While SERCA2b regulates basal $\left[\mathrm{Ca}^{2+}\right]^{c 46}$, it has been suggested that SERCA3 is responsible for replenishing $\mathrm{ER} \mathrm{Ca}^{2+}$ when the cytosolic $\mathrm{Ca}^{2+}$ level is elevated by depolarization ${ }^{47}$, thus prolonging the $\mathrm{Ca}^{2}$ oscillation. We showed that overexpression of BNLN did not alter $\mathrm{KCl}$-induced $\left[\mathrm{Ca}^{2+}\right]^{\mathrm{c}}$ peak, but decreased $\left[\mathrm{Ca}^{2+}\right]^{\mathrm{ER}}$ and increased $\left[\mathrm{Ca}^{2+}\right]^{\mathrm{C}}$ in response to stimulatory glucose concentrations (Fig. 4E). This is in line with a study from Ravier et al., which showed that SERCA3-null islets exhibited decreased ER $\mathrm{Ca}^{2+}$ influx, 41 leading to an increased cytosolic $\mathrm{Ca}^{2+}$ oscillatory amplitude and enhanced insulin secretion ${ }^{48}$. Our study 
42 mainly relied on INS-1 cells as a surrogate for $\beta$-cells, and determining how BNLN modulates islet $43 \mathrm{Ca}^{2+}$ oscillation/kinetics in health and obesity/diabetes requires further studies.

We found that, in $\beta$-cells, BNLN interacts with SERCA3A (Fig. $4 \mathrm{H} \& \mathrm{I}$ ), and decreases $\mathrm{Ca}^{2+}$ buffer capacity of the ER in response to high glucose (Fig. 4A\&B), indicating a potential inhibitory role of BNLN on SERCA3. However, whether this is primarily the result of reduction of SERCA3A enzyme activity requires further investigation. Furthermore, sustained high-glucose elicits ER stress in the $\beta$-cell ${ }^{35}$. Disturbed ER homeostasis leads to impaired $\mathrm{ER} \mathrm{Ca}^{2+}$ efflux, $\mathrm{Ca}^{2+}$-induced $\mathrm{Ca}^{2+}$ release $(\mathrm{CICR})$, as well as dysfunction of insulin production in the $\beta$-cell. We found that the high glucose-induced ER stress was ameliorated by

BNLN overexpression, while BNLN decreased ER Ca" level. Further study is needed to comprehensively delineate the impact of BNLN on ER homeostasis and ER Ca" leakage in $\beta$-cells under diverse ER stress conditions. Notably, glucose and amino acids promote ER Ca ${ }^{2+}$ uptake in the absence of external $\mathrm{Ca}^{2+37,49}$, suggesting that cytosolic $\mathrm{Ca}^{2+}$-binding proteins and other intracellular $\mathrm{Ca}^{2+}$ storage represent an important source of $\mathrm{Ca}^{2+}$ that can be pumped by the ER. Future study will be required to establish the functional impact of BNLN on other protein binding partners (Supplemental Table 2).

In summary, our study demonstrates that TUNAR, a previously annotated IncRNA, encodes a conserved 48aa micropeptide BNLN, which modulates $\mathrm{Ca}^{2+}$ homeostasis in $\beta$-cells, consequently regulating GSIS. This study indicates that dysregulation of BNLN in the pancreas might contribute to the obesity-associated malfunction of insulin secretion. Our study provides important insights into the molecular mechanisms that underlie the fine-tuning of glucose-induced insulin secretion by translational function of IncRNA. In addition, our study will also have a broader impact on understanding of the interactions between IncRNA biology and dysfunction of islets relevant to obesity and type 2 diabetes. It remains an intriguing possibility that insights from this study could be exploited for therapeutic interventions in obesity and type 2 diabetes. 
M.L. and F.S performed the experiments, analyzed the data. QW.Q, WJ.Y., ZY.Z., BY.C, D.S., YW.G., and

AV.P. performed the experiments. J.S., Y.I, S.S. and LS.S. provided critical reagents and scientific

suggestions on this study. HJ.C and L.Y conceived and supervised the study and wrote the manuscript.

L.Y. is supported by NIH (DK108835-01A1) and American Diabetes Association (1-18-IBS-149); Z.Z. is

supported by American Heart Association (19PRE34380258).

\section{ACKNOWLEDGEMENT}

We are grateful to the Alberta Diabetes Institute Islet Core (Canada) for providing us the human islets, and

the contributions of the donors whose islets were used, as well as the physicians, nurses, and researchers

who procured the specimens. We also thank Dr. Michael Welsh (University of lowa) for providing technical

supports in RNA imaging; Dr. Monica Nagendran and Dr. Tushar Desai (Stanford University School of

Medicine) for sharing the Proximity ligation in situ hybridization protocol with Welsh lab. We thank Dr.

Timothy Ryan (Weill Cornell Medicine) for donating the ER-GCaMP6-15 to Addgene and Alyona Li for

81 technical support with graphic illustrations. We would like thank members of the Yang, Cao, and Amendt 82 laboratories for helpful discussion and suggestions.

\section{MATERIAL AND METHODS}

85 Prioritize putative micropeptide encoding IncRNAs in human pancreas

86 RNA-seq data of human pancreas islets were downloaded from EBI arrayexpress database (E-MTAB$871^{1294^{34}}$ ). RNA-seq reads were quality checked using the FastQC tool 88 (http://www.bioinformatics.babraham.ac.uk/projects/fastqc). Low-quality and adapter sequences were 89 trimmed using the Trimmomatic ${ }^{50}$. Expression of transcripts was quantified using the Salmon ${ }^{51}$, and evaluated for their coding potential with PhyloCSF and RNAcode. We downloaded 100-species whole 
and prepare multispecies DNA sequence alignments for each transcript of protein coding (<100aa) genes

and IncRNAs. We filtered for transcripts that had at least 2 species that have more than 60 -nucleotide (nt)

DNA sequence in the multispecies alignments. PhyloCSF and RNAcode were run with these multispecies

DNA sequence alignments as input. To prioritize search of putative micropeptide encoded by IncRNAs, we ranked genes with both expression level in human islets and coding potential.

$\underline{\text { Animals }}$

Animal care and experimental procedures were performed with approval from the University of lowa's Institutional Animal Care and Use Committee. C57BL/6J mice (The Jackson Laboratory, 000664) were

kept on a $12 \mathrm{hr}$ light/dark cycle, and were fed the RD (7319 Teklad global diet). Mice used in generating

the DIO model were placed on a 60\% kcal high-fat diet (HFD; Research Diets, D12492) immediately after weaning, at 3 weeks of age.

06

\section{$\underline{\text { Human islets }}$}

08 Human islet study was reviewed by IRB at University of lowa and deemed non-human study. Human islets were received from the Alberta Diabetes Institute Islet Core with reported viability and purity above $80 \%$. Islet were cultured in CMRL1066 (Thermo Fisher Scientific, 11530037) medium with 1\% human serum albumin, 1\% Pen-Strep (Thermo Fisher Scientific, 15140163), and 1\% L-Glutamine (Thermo Fisher

Scientific, 25030164) overnight at $37^{\circ} \mathrm{C}$ upon arrival for recovery upon shipping. The eight donors were:

age, 20-69 years; BMI, 19-33.9; Hba1c, 4.9-5.8; males; cause of death, neurological.

Cell culture, transfection and Luciferase Assay

The rat insulinoma cells (INS-1, 832/3) ${ }^{54}$ were cultured in RPMI-1640 medium (Thermo Fisher Scientific, 11875119) supplemented with 10\% FBS, 10mmol/L HEPES (Thermo Fisher Scientific, 15630130), 2mmol/L L-glutamine, 1 mmol/L sodium pyruvate (Sigma-Aldrich, P2256), and 50 $\mathrm{mol} / \mathrm{L}$ 2-mercaptoethanol (VWR, 97064-880). Cells were transfected with DNA constructs (1ug/well in 12-well plate) using 
21 31985088). At $48 \mathrm{hr}$ post transfection, the transfected cells were trypsinized (Trypsin-EDTA 0.05\%; Thermo

Fisher Scientific, 25300054), mixed, and re-plated in an appropriate plate in order to achieve homogeneous

population across wells subsequent analyses. The HEK 293T cells (ATCC) were cultured in DMEM

medium with $10 \%$ FBS, $1 \%$ penicillin-streptomycin. Cells were transfected with DNA constructs $(0.5$ ug/well

of 24-well plate) using Lipofectamine 3000 reagent (Thermo Fisher, L3000015) in Opti-MEM medium

(Thermo Fisher Scientific, 31985-062). At $48 \mathrm{hr}$ post transfection, the activities of firefly luciferase and

Renilla luciferase were measured using the Luciferase Assay System (Promega, E1500) and Renilla

Luciferase Assay System (Promega, E2810), separately.

\section{Constructs, adenovirus preparation and transduction}

31 The BNLN constructs were generated by Sequence- and ligation-independent cloning (SLIC) method ${ }^{55}$. 32 The detailed information of constructs used in each figure is list in supplementary Table2. Briefly, tRFP and 33 Renilla luciferase-GFP expressing sequences were fused to C-terminus of human BNLN (in-frame and out34 of-frame; fs1, fs2). The recombined fragments were then inserted into pCAGEN vector (Addgene, 11179). 35 To determine transcription initial site and BNLN localization, full length human BNLN, ATGs- and CTG36 deleted BNLN (dATG1, dATG2, dATG3, dCTG) were fused with Flag and cloned into pcDNA3.1(-) 37 (Invitrogen, V79520) vector. KDEL plasmid was obtained from Addgene (114177). The HiBiT-BNLN and V5-BNLN constructs were generated by fusing HiBiT vector (Promega) and V5 sequence, respectively, inframe with N-terminus of BNLN. The fused fragments were then inserted into PQCXIP vector (Addgene, 631516), by using NEBuilder® HiFi DNA Assembly Cloning Kit (New England Biolabs, E5520S). ER-GCaMP6-15 $15^{56}$ was provided by Addgene. Adenoviruses carrying mouse TUNA BNLN were generated using the ViraPowerTM adenoviral expression system (Invitrogen). Adenoviruses were amplified in HEK293A cells (ATCC) and purified by CsCl gradient centrifugation. The viruses were titered using AdenoX Rapid Titer Kit (Takaba Bio, 632250) and transduced into the cells as previously described ${ }^{57}$. Cultured intact mouse or human islets were resuspended in $0.1 \mathrm{mmol} / \mathrm{L}$ EGTA (Sigma-Adlrich, 234626) in serum free CRMLfollowed by transduction of indicated adenoviral constructs at 10,000 plaque-forming unit per 
48 inactivated FBS for culture overnight[]. The culture medium was replaced next day and the islets were cultured for additional $24 \mathrm{hr}$ before subjecting them to glucose stimulated insulin secretion (GSIS) assay.

Nano-Glo HiBiT extracellular detection system

Ins-1 cells were transfected with FLAG-GFP or HiBiT-BNLN as described above and seeded in an opaque 96-well plate(Fisher Scientific, 08-771-26) at $\sim 15,000$ cells/well in the culture medium. The experiment

was carried out according to the manufacturer's protocol (Promega, N2422). Briefly, the transfected cells

were incubated with Nano-Glo HiBiT extracellular buffer, LgBiT protein, and Nano-Glo HiBiT extracellular

substrate, and luminescence was detected after 5-10min using a luminescence microplate reader

(SpectraMax, Molecular Devices, USA). The expression of extracellular HiBiT-BNLN was normalized to

the total intracellular pool of HiBiT-BNLN by lysing the cells and measuring luminescence with Nano-Glo HiBiT lytic detection system (Promega, N2422), following the protocol.

$\underline{\text { Western Blotting and immunoprecipitation }}$

62 Cells were lysed with a lysis buffer containing $0.4 \%$ of NP-40, protease inhibitors cocktail (Sigma-Aldrich, P8340), and $\mathrm{Na}_{3} \mathrm{VPO}_{4}$ (Sigma-Aldrich). Protein concentration was determined by Pierce BCA kit (Thermo

Fisher Scientific, 23225) and samples were subjected to SDS-polyacrylamide gel electrophoresis, as previously described (48). For immunoprecipitation assay, cells were lysed in radioimmunoprecipitation assay (RIPA) buffer (50mM Tris, $150 \mathrm{mM} \mathrm{NaCl}, 1 \% \mathrm{NP} 40,0.25 \%$ sodium deoxycholate). $1 \mathrm{mg}$ proteins were immunoprecipitated with protein A/G magnetic Beads (ThermoFisher, 88802) conjugated with anti-IgG (Cell Signaling Technology, 2729), anti-Flag (Thermo Fisher, A36797) and eluted by $4 \times$ lituium dodecyl sulfate (LDS; Invitrogen, NP0008). Membranes were immunoblotted with anti-V5 (ab15528), anti-FLAG and anti-beta Actin (ab8227) at a 1:1000 dilution. Secondary antibodies were horseradish peroxidaseconjugated goat-anti-mouse-lgG (Santa Cruz Biotechnology, sc-2005), horseradish peroxidaseconjugated mouse-anti-rabbit-lgG (Santa Cruz Biotechnology, sc-2357) at a 1:10000 dilution. Signal was detected using the ChemiDoc Touch Imaging System (BioRad), and densitometry analyses of western blot 74 images were performed by using the Image Lab software (BioRad). 
$76 \quad$ Proximity ligation in situ hybridization (PLISH)

77 In situ hybridization was performed as previously described ${ }^{58}$ with some modifications. Briefly, human islets were fixed and sectioned following the typical formalin-fixed paraffin embedded tissue processing protocol.

The sections were then incubated in citrate-based target unmasking solution (Vector laboratories, $\mathrm{H}-3300$ ) with $0.05 \%$ lithium dodecyl sulfate (Sigma, L4632) at $65^{\circ} \mathrm{C}$ for $30 \mathrm{~min}$. The slides were then incubated with $0.05 \mathrm{mg} / \mathrm{ml}$ pepsin (Sigma-Aldrich, P6887) in $0.1 \mathrm{M} \mathrm{HCl}$ for $10 \mathrm{~min}$ at $37^{\circ} \mathrm{C}$, and processed with the typical proximity ligation in situ hybridization ${ }^{58}$. RNA sequences that were targeted by short-paired hybridization probes were: Human INS-1: CTGGTGGAAGCTCTCTACCTAGTGTGCGGGGAACGAGGCT;Human INS-

2: GCTGGAGAACTACTGCAACTAGACGCAGCCCGCAGGCAGC; HumanGCG-1: TGGACT

CCAGGCGTGCCCAAGATTTTGTGCAGTGGTTGAT; Human GCG-2: AGATGAACACCATTCTTGATAA

TCTTGCCGCCAGGGACTT; Human GCG-3: TCTTCACAACATCACCTGCTAGCCACGTGGGATGTTTG

AA; Human BNLN: TCTTGGCAATGCTGGGGATTATCGGGACCATTCTGAACCT; Human BNLN: AGCA

GGCTTGACCCGCACATACCACCCAATCAAATGCACC'; Human BNLN: ATTCCAGATCGCTGACAGAT

ATCACATATTTGAAAAGATG; Human BNLN: CTTCACTTGACGAGCTATTTAGTGGAAAAACCACAGG

CGC; Human BNLN: GCAGACCTTAGATGCACCCTATCTTTACTGAGAATTATGC; Human BNLN:

\section{TTTGATTTTAGCGGTCATGTACCGCGAGAGTTGGGAAGAA.}

Immunocytochemistry and Confocal microscopy

INS-1 cells were then fixed in $4 \%$ paraformaldehyde for $10 \mathrm{~min}$ at room temperature, blocked in $5 \%$ goat serum, incubated with Hoechst33342 (Thermo Fisher Scientific, H3570), and mounted with a glass coverslip. For subcellular organelle localization, Lystroacker ${ }^{\mathrm{TM}}$ Red DND-99 (Thermo Fisher Scientific, L7528), Mitotracker ${ }^{\mathrm{TM}}$ Red CMXRos (Thermo Fisher Scientific, M7512), ACAA1 antibody (Gene Tex, GTX114229), RFP-KDEL plasmid, and TGN38 antibody (Novus, NB300-575) were used to detect lysosomes, mitochondria, peroxisomes, the ER, and Golgi respectively. Images were acquired using a 
02 different channels were merged. For PLISH assays, stack images were taken using the $60 \mathrm{x}$ lens of 03 Olympus microscopy (FV3000, confocal laser scanning microscope).

.05 Quantitative real-time RT-PCR

Total RNA was isolated using the Trizol reagent (Invitrogen, 15596026) and reverse transcribed into cDNA

using a PrimeScript RT RT Master Mix (Takara RR036A). Quantitative real-time RT-PCR analysis was

performed using TB Green Premix Ex Taq (Clontech, RR420A) and following primers: BNLN-F: 5'-TTC

TCC TCG CCT TCC TGC-3'; BNLN-R: 5'-CAT CTT GGT TGC AAA TGT CT-3'; ACTB-F: 5'-CTC TTC CAG

CCT TCC TTC-3'; ACTB-R: 5'-ATC TCC TTC TGC ATC CTG TC-3'; Hprt-F: 5'-CAG TCC CAG CGT CGT

GAT TA-3'; Hprt-R: 5'-GGC CTC CCA TCT CCT TCA TG-3'; Bnln-F: 5'-GGA GAA TGA GGC AGG AAC

CC-3'; Bnln-R: 5'ACA CGC TCT CTT CCT TGC TC-3'.

$14 \quad$ Calcium imaging

$15\left[\mathrm{Ca}^{2+}\right]^{\mathrm{c}}$ measurement: INS-1 832/3 cells were transfected with FLAG-GFP or FLAG-GFP-BNLN. 24hr after .16 transfection, the Ins-1 cells were plated at $\sim 25,000$ cells/well in a HTB9-coated Greiner Sensoplate ${ }^{\mathrm{TM}}$ glass 17 bottom 96-well plate (Millipore Sigma). Prior to imaging, the cells were loaded with Screen Quest ${ }^{\mathrm{TM}}$ Fluo.188 no wash calcium red cye (AAT Bioquest, 36314) indicator in the DMEM (Thermo Fisher Scientific, .19 A1443001) culture medium supplemented with $2.5 \mathrm{mM}$ glucose and $0.1 \%$ BSA for $1 \mathrm{hr}$ at $37^{\circ} \mathrm{C}$. A fluorescent microplate reader (CLARIOstar PLUS, BMG Labtech, Germany) was used to inject glucose 21 (final concentration of $20 \mathrm{mM}$ ) to cells, and to acquire fluorescent signal every $0.6 \mathrm{sec}$ for $10 \mathrm{~min}$. Devices, USA), INS-1 cells were transfected with pQCXIP, FLAG-BNLN, or V5-BNLN (1ug DNA/well) in a 12-well plate (3 wells/each DNA construct) with Lipofectamine 2000, as described above. A suspension of Ins-1 cells was pipetted in a 384-well culture black microplate with optic bottom plate (Greiner-Bio, 781090) at $\sim 17,000$ cells/well in $25 \mathrm{uL}$ of the low glucose DMEM culture medium. $25 \mathrm{uL}$ of Fluo-8 calcium dye was used added to the cell suspension. The plate was incubated at $37^{\circ}$ for $30 \mathrm{~min}$, followed by another $30 \mathrm{~min}$ 
with low glucose $(27.5 \mathrm{mM})$, high glucose $(181.5 \mathrm{mM})$, and potassium chloride as a positive control $(220 \mathrm{mM})$.

The FLIPR was utilized to transfer the solutions from the compound plate into the black plate and to read

the $\mathrm{Ca}^{2+}$ signal. The basal signal was measured for 20 seconds every second, then the solutions were added (5uL/each), and the response signal was read for $10 \mathrm{~min}$ every $0.8 \mathrm{sec}$.

$\left[\mathrm{Ca}^{2+}\right]^{E R}$ measurement: INS-1 cells were co-transfected with ER-GCaMP6s and pQCXIP or V5-BNLN using

Lipofectamine 2000 , as described above. Next day, the cells were plated at $\sim 25,000$ cells/well in a HTB-9

coated plate, as described above. 24hours later, the cells were subjected to treatment with DMEM

containing $2.5 \mathrm{mM}$ glucose at $37^{\circ}$ for $1 \mathrm{hr}$. Basal signal was acquired in the GFP excitation/emission spectrum using the fluorescent microplate reader as described above every $0.8 \mathrm{sec}$ for $1 \mathrm{~min}$. Then a high glucose solution ( $17 \mathrm{mM}$ final concentration) was injected and the fluorescent signal was collected for $5 \mathrm{~min}$.

Murine islet isolation

41 The islets were isolated as previously described ${ }^{59}$. Briefly, lean C57BL/6J male mice (12-20 weeks of age) and mice with diet-induced obesity (12-16 weeks of HFD) were anesthetized with isoflurane, and the pancreas was perfused with HBSS containing type $V$ collagenase $(0.8 \mathrm{mg} / \mathrm{mL})$ via the common bile duct. The pancreas was then removed and digested at $37^{\circ} \mathrm{C}$ for $10-12$ min with physical agitation to release islets. Islets were further washed with RPMI-1640 with 1\% FBS and purified on Histopaque 1077 and 1119 gradients Sigma-Aldrich, 10771, 11191). The islets were then collected, washed, and cultured in RPMI1640 supplemented with $10 \%$ FBS, $1 \%$ pen-strep, and $1 \%$ HEPES in $30 \mathrm{~mm}$ dishes at $37^{\circ} \mathrm{C}$.

Glucose stimulated insulin secretion (GSIS)

For GSIS in murine islets, 30-40 islets of similar size were picked and placed in separate wells in a plastic 12-well plate (Fisher Scientific, 07-200-82). The islets were rinsed with $1 \mathrm{~mL}$ of Secretion Assay Buffer (SAB) adjusted to $\mathrm{pH} 7.2$, containing $0.114 \mathrm{~mol} / \mathrm{L}$ sodium chloride (Sigma Aldrich, S9888), $0.47 \mathrm{mmol} / \mathrm{L}$ potassium chloride (Sigma Aldrich, P9333), 0.12mmol/L potassium dihydrogen phosphate (Sigma Aldrich, 1.04871), 0.116mmol/L magnesium sulfate (Sigma Aldrich, M2643), 20mmol/L HEPES (Thermo Fisher Scientific, 15630130), 2.5mmol/L calcium chloride (Sigma Aldrich, 449709), 0.2\% bovine serum albumin, and $0.2 \%$ 
56 sodium bicarbonate (Sigma Aldrich, S5761) ${ }^{59}$. The islets were then equilibrated in SAB containing low .57 glucose $(2.5 \mathrm{mmol} / \mathrm{L})$ for $1 \mathrm{hr}$ at $37^{\circ} \mathrm{C}$. Next, the islets were incubated in fresh SAB with low glucose for additional $1-2$ hours at $37^{\circ} \mathrm{C}$. The supernatant was collected for measuring basal insulin secretion. The islets were then incubated with $\mathrm{SAB}$ containing high glucose $(16.7 \mathrm{mmol} / \mathrm{L})$ for $1-2$ hours at $37^{\circ} \mathrm{C}$. The supernatant was collected for measuring glucose-stimulated insulin secretion. The islets were lysed in acidified ethanol and insulin content was measured. Secreted insulin and insulin content were analyzed with STELLUX® Chemi Rodent Insulin ELISA (ALPCO, 80-INSMR-CH01), according to the manufacturer's protocol.

For GSIS in Human islets, 30-40 islets of similar size were picked and subjected to GSIS similar to murine islets. Secreted insulin and insulin content were analyzed with STELLUX® Chemi Human Insulin ELISA (ALPCO, 80-INSHU-CH01), according to the manufacturer's protocol.

GSIS in Ins-1 cells: $100 \%$ confluent cells were rinsed with SAB and equilibrated with low-glucose-SAB for

1-2 hours at $37^{\circ} \mathrm{C}$. Fresh SAB containing low glucose was added to the cells for additional 1-2 hours at

$37^{\circ} \mathrm{C}$. The supernatant was then collected for measuring basal insulin secretion. SAB containing high

glucose was added to the cells and incubated for additional $1-2$ hours at $37^{\circ} \mathrm{C}$ before supernatant collection.

The cells were lysed and protein was extracted for insulin content. Secreted insulin and insulin content were analyzed with STELLUX® Chemi Rodent Insulin ELISA (ALPCO), according to the manufacturer's protocol.

$\underline{\text { Statistics }}$

The data are presented as mean \pm standard error of the mean or standard deviation as noted; $n$ represents the number of individual experiments; $N$ represents the number of human donors or mice. Statistical differences of numeric parameters between two groups were determined with Student's $t$-test. For 81 statistical analysis of differences between multiple groups, one way and two-way ANOVA were applied, 82 followed by post-hoc Tukey's test. The statistical analyses were performed in Prism. 


\section{REFERENCES}

1. Forrest, A.R.R., et al. A promoter-level mammalian expression atlas. Nature 507, 462-470 (2014).

2. Davis, C.A., et al. The Encyclopedia of DNA elements (ENCODE): data portal update. Nucleic Acids Res 46, D794-D801 (2018).

3. Dunham, I., et al. An integrated encyclopedia of DNA elements in the human genome. Nature 489, 57-74 (2012).

4. Hon, C.-C., et al. An atlas of human long non-coding RNAs with accurate 5' ends. Nature 543, 199204 (2017).

5. Akerman, I., et al. Human Pancreatic beta Cell lncRNAs Control Cell-Specific Regulatory Networks. Cell Metab 25, 400-411 (2017).

6. Moran, I., et al. Human beta cell transcriptome analysis uncovers lncRNAs that are tissue-specific, dynamically regulated, and abnormally expressed in type 2 diabetes. Cell Metab 16, 435-448 (2012).

7. Wong, W.K.M., Sorensen, A.E., Joglekar, M.V., Hardikar, A.A. \& Dalgaard, L.T. Non-Coding RNA in Pancreas and beta-Cell Development. Noncoding RNA 4(2018).

8. Alvarez, M.L. \& DiStefano, J.K. Functional characterization of the plasmacytoma variant translocation 1 gene (PVT1) in diabetic nephropathy. PLoS One 6, e18671 (2011).

9. Carter, G., et al. Circulating long noncoding RNA GAS5 levels are correlated to prevalence of type 2 diabetes mellitus. BBA Clin 4, 102-107 (2015).

10. de Gonzalo-Calvo, D., et al. Circulating long-non coding RNAs as biomarkers of left ventricular diastolic function and remodelling in patients with well-controlled type 2 diabetes. Sci Rep 6, 37354 (2016).

11. Makarewich, C.A. \& Olson, E.N. Mining for Micropeptides. Trends in Cell Biology 27, 685-696 (2017).

12. Anderson, D.M., et al. A Micropeptide Encoded by a Putative Long Noncoding RNA Regulates Muscle Performance. Cell 160, 595-606 (2015).

13. Anderson, D.M., et al. Widespread control of calcium signaling by a family of SERCA-inhibiting micropeptides. Sci. Signal. 9, ra119-ra119 (2016).

14. Cai, B., et al. LncRNA-Six1 Encodes a Micropeptide to Activate Six1 in Cis and Is Involved in Cell Proliferation and Muscle Growth. Front. Physiol. 8(2017).

15. D'Lima, N.G., et al. A human microprotein that interacts with the mRNA decapping complex. Nature Chemical Biology 13, 174-180 (2017).

16. Makarewich, C.A., et al. MOXI Is a Mitochondrial Micropeptide That Enhances Fatty Acid $\beta$ Oxidation. Cell Reports 23, 3701-3709 (2018).

17. Matsumoto, A., et al. mTORC1 and muscle regeneration are regulated by the LINC00961-encoded SPAR polypeptide. Nature 541, 228-232 (2017).

18. Nelson, B.R., et al. A peptide encoded by a transcript annotated as long noncoding RNA enhances SERCA activity in muscle. Science 351, 271-275 (2016).

19. Pauli, A., et al. Toddler: An Embryonic Signal That Promotes Cell Movement via Apelin Receptors. Science 343, 1248636 (2014).

20. Stein, C.S., et al. Mitoregulin: A lncRNA-Encoded Microprotein that Supports Mitochondrial Supercomplexes and Respiratory Efficiency. Cell Reports 23, 3710-3720.e3718 (2018).

21. Pauli, A., et al. Toddler: an embryonic signal that promotes cell movement via Apelin receptors. Science 343, 1248636 (2014).

22. Lee, C., et al. The mitochondrial-derived peptide MOTS-c promotes metabolic homeostasis and reduces obesity and insulin resistance. Cell Metab 21, 443-454 (2015).

23. Makarewich, C.A., et al. MOXI Is a Mitochondrial Micropeptide That Enhances Fatty Acid betaOxidation. Cell Rep 23, 3701-3709 (2018). 
24. Lin, M.F., Jungreis, I. \& Kellis, M. PhyloCSF: a comparative genomics method to distinguish protein coding and non-coding regions. Bioinformatics 27, i275-282 (2011).

25. Washietl, S., et al. RNAcode: robust discrimination of coding and noncoding regions in comparative sequence data. RNA 17, 578-594 (2011).

26. Fu, Z., Gilbert, E.R. \& Liu, D. Regulation of insulin synthesis and secretion and pancreatic Betacell dysfunction in diabetes. Curr Diabetes Rev 9, 25-53 (2013).

27. Araki, E., Oyadomari, S. \& Mori, M. Impact of endoplasmic reticulum stress pathway on pancreatic beta-cells and diabetes mellitus. Exp Biol Med (Maywood) 228, 1213-1217 (2003).

28. Berridge, M.J. The endoplasmic reticulum: a multifunctional signaling organelle. Cell Calcium 32, 235-249 (2002).

29. Zhang, I.X., Raghavan, M. \& Satin, L.S. The Endoplasmic Reticulum and Calcium Homeostasis in Pancreatic Beta Cells. Endocrinology 161(2020).

30. Engin, F., Nguyen, T., Yermalovich, A. \& Hotamisligil, G.S. Aberrant islet unfolded protein response in type 2 diabetes. Sci Rep 4, 4054 (2014).

31. Safra, M., Ben-Hamo, S., Kenyon, C. \& Henis-Korenblit, S. The ire-1 ER stress-response pathway is required for normal secretory-protein metabolism in C. elegans. Journal of cell science 126, 41364146 (2013).

32. Akerman, I., et al. Human Pancreatic $\beta$ Cell lncRNAs Control Cell-Specific Regulatory Networks. Cell Metabolism 25, 400-411 (2017).

33. Lin, N., et al. An Evolutionarily Conserved Long Noncoding RNA TUNA Controls Pluripotency and Neural Lineage Commitment. Molecular Cell 53, 1005-1019 (2014).

34. Morán, I., et al. Human $\beta$ Cell Transcriptome Analysis Uncovers lncRNAs That Are Tissue-Specific, Dynamically Regulated, and Abnormally Expressed in Type 2 Diabetes. Cell Metabolism 16, 435448 (2012).

35. Arruda, A.P. \& Hotamisligil, G.S. Calcium Homeostasis and Organelle Function in the Pathogenesis of Obesity and Diabetes. Cell Metab 22, 381-397 (2015).

36. Fu, S., et al. Phenotypic assays identify azoramide as a small-molecule modulator of the unfolded protein response with antidiabetic activity. Science translational medicine 7, 292ra298 (2015).

37. Gilon, P., Chae, H.Y., Rutter, G.A. \& Ravier, M.A. Calcium signaling in pancreatic beta-cells in health and in Type 2 diabetes. Cell Calcium 56, 340-361 (2014).

38. Batista, P.J. \& Chang, H.Y. Long Noncoding RNAs: Cellular Address Codes in Development and Disease. Cell 152, 1298-1307 (2013).

39. Sarropoulos, I., Marin, R., Cardoso-Moreira, M. \& Kaessmann, H. Developmental dynamics of lncRNAs across mammalian organs and species. Nature 571, 510-514 (2019).

40. Angcajas, A.B., et al. Diversity of amino acid signaling pathways on autophagy regulation: a novel pathway for arginine. Biochemical and biophysical research communications 446, 8-14 (2014).

41. Ulitsky, I., Shkumatava, A., Jan, C.H., Sive, H. \& Bartel, D.P. Conserved function of lincRNAs in vertebrate embryonic development despite rapid sequence evolution. Cell 147, 1537-1550 (2011).

42. Dai, J., Ma, J., Yu, B., Zhu, Z. \& Hu, Y. Long Noncoding RNA TUNAR Represses Growth, Migration, and Invasion of Human Glioma Cells Through Regulating miR-200a and Rac1. Oncol Res 27, 107-115 (2018).

43. Makarewich, C.A. The hidden world of membrane microproteins. Exp Cell Res 388, 111853 (2020).

44. Bertram, R. \& Sherman, A. Filtering of calcium transients by the endoplasmic reticulum in pancreatic beta-cells. Biophys $J$ 87, 3775-3785 (2004).

45. Misquitta, C.M., Mack, D.P. \& Grover, A.K. Sarco/endoplasmic reticulum Ca2+ (SERCA)-pumps: link to heart beats and calcium waves. Cell Calcium 25, 277-290 (1999).

46. Klionsky, D.J., et al. Guidelines for the use and interpretation of assays for monitoring autophagy (3rd edition). Autophagy 12, 1-222 (2016). 
47. Arredouani, A., et al. SERCA3 ablation does not impair insulin secretion but suggests distinct roles of different sarcoendoplasmic reticulum $\mathrm{Ca}(2+)$ pumps for $\mathrm{Ca}(2+)$ homeostasis in pancreatic betacells. Diabetes 51, 3245-3253 (2002).

48. Ravier, M.A., et al. Mechanisms of control of the free $\mathrm{Ca} 2+$ concentration in the endoplasmic reticulum of mouse pancreatic beta-cells: interplay with cell metabolism and $[\mathrm{Ca} 2+] \mathrm{c}$ and role of SERCA2b and SERCA3. Diabetes 60, 2533-2545 (2011).

49. Zarain-Herzberg, A., Garcia-Rivas, G. \& Estrada-Aviles, R. Regulation of SERCA pumps expression in diabetes. Cell Calcium 56, 302-310 (2014).

50. Bolger, A.M., Lohse, M. \& Usadel, B. Trimmomatic: a flexible trimmer for Illumina sequence data. Bioinformatics 30, 2114-2120 (2014).

51. Patro, R., Duggal, G., Love, M.I., Irizarry, R.A. \& Kingsford, C. Salmon provides fast and biasaware quantification of transcript expression. Nat Methods 14, 417-419 (2017).

52. Soneson, C., Love, M.I. \& Robinson, M.D. Differential analyses for RNA-seq: transcript-level estimates improve gene-level inferences. F1000Res 4, 1521 (2015).

53. Gentleman, R.C., et al. Bioconductor: open software development for computational biology and bioinformatics. Genome Biol 5, R80 (2004).

54. Hohmeier, H.E., et al. Isolation of INS-1-derived cell lines with robust ATP-sensitive K+ channeldependent and -independent glucose-stimulated insulin secretion. Diabetes 49, 424-430 (2000).

55. Beigi, F., et al. Dynamic denitrosylation via S-nitrosoglutathione reductase regulates cardiovascular function. Proceedings of the National Academy of Sciences of the United States of America 109, 4314-4319 (2012).

56. de Juan-Sanz, J., et al. Axonal Endoplasmic Reticulum $\mathrm{Ca}(2+)$ Content Controls Release Probability in CNS Nerve Terminals. Neuron 93, 867-881 e866 (2017).

57. Yang, L., et al. METABOLISM. S-Nitrosylation links obesity-associated inflammation to endoplasmic reticulum dysfunction. Science 349, 500-506 (2015).

58. Nagendran, M., Riordan, D.P., Harbury, P.B. \& Desai, T.J. Automated cell-type classification in intact tissues by single-cell molecular profiling. Elife 7(2018).

59. Stephens, S.B., et al. A VGF-derived peptide attenuates development of type 2 diabetes via enhancement of islet beta-cell survival and function. Cell Metab 16, 33-43 (2012).

\section{FIGURE LEGENDS}

Figure 1. Identification of IncRNAs that have coding potential in the human pancreas. A. Scott plot of IncRNA expression level in human islets and coding potential. Expression level was measured by $\log 2(T P M+1)$. Coding potential was measured by log2(PhyloCSF score + 1). B. PhyloCSF score and RNAcode score for 12 IncRNAs that have recently been found to encode micropeptides. TUNAR is the micropeptide encoding IncRNA identified in this study. C. Genomic architecture of the TUNAR locus. 100 vertebrates sequence conservation shows a conserved region at the beginning of last exon. D. Multispecies alignment of predicted TUNAR encoding micropeptide BNLN. A conserved transmembrane domain is located at the C-terminus. Three potential start codons were presented. 
Figure 2. TUNAR encodes a novel micropeptide, BNLN. A. Schematic representation of BNLN reporter

constructs. BNLN coding sequence (CDS) is shown in pink block. B. Representative images (20X) and C.

Activity of Renilla luciferase in HEK 293T cells transfected with the indicated BNLN constructs as in (A) for $48 \mathrm{hr}$. Scale bar: $50 \mu \mathrm{m}$. The data were normalized to firefly luciferase. * indicates statistical significance compared to in-frame constructs ( $n=4$, biological replicates). $A U$, arbitrary units.

D. Representative western blots of BNLN expression in HEK 293T cells transfected with the indicated BNLN constructs. CT: nontransfected cell control. E. Representative western blots of BNLN expression in cells transfected with the indicated BNLN constructs for 48hrs. F. Levels of mRNAs encoding for BNLN in HEK293T cells expressing full length, ATG-deleted and CTG-deleted BNLN CDS. The data were normalized to ACTB expression level. * indicates statistical significance compared to the full $B N L N$ expressing cells ( $\mathrm{n}=6$, biological replicates). Data are shown as means \pm SEM, statistical significance was determined by ANOVA followed by Sidak's multiple comparisons test in C\&F. $p<0.05$.

Figure 3. BNLN localizes at the endoplasmic reticulum in $\boldsymbol{\beta}$-cells. A. Representative confocal images (60X) of TUNAR in human islets. Small molecular in situ hybridization of BNLN, and INSULIN ( $\beta$-cell marker). Scale bar: $10 \mu \mathrm{m}$. B. Representative western blots of BNLN expression in INS-1 cells transfected with the indicated BNLN constructs for 48hrs. C. Representative confocal images (63X) of BNLN in INS-1 cells co-transfected with FLAG-GFP-BNLN and KDEL-RFP (24hrs post transfection). CT vevtor: FLAGGFP construct. Scale bar: $10 \mu \mathrm{m}$. D. Representative confocal images (63X) of BNLN in INS-1 cells transfected with BNLN-GFP and stained with lysotracker (lysosomes), mitotracker (mitochondria), Acaa1

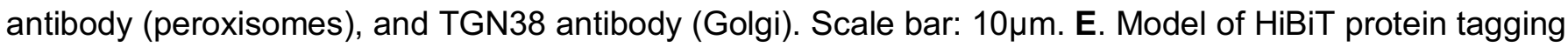
system in monitoring BNLN localization. INS-1 cells were transfected with HiBiT-tagged BNLN, the cell surface HiBiT was recognized by recombinant LgBiT and NanoBiT substrate in live cells. F. Expression of cell surface HiBiT-BNLN level in INS-1 cells $24 \mathrm{hrs}$ after transfecting with the HiBiT-BNLN construct. The level of cell surface HiBiT-BNLN was measured by using LgBiT and the extracellular substrate, and 
of LgBiT. Data are shown as means \pm SEM, and statistical significance was determined by Student's t-test $(p<0.05, n=3$, biological replicates). AU, arbitrary units.

Figure 4. BNLN regulates glucose-induced calcium dynamics in pancreatic $\beta$-cells. A.

Representative ER Ca ${ }^{2+}$ recording of INS-1 cells cotransfected with V5-BNLN and an ER calcium probe (GCaMP6s) after stimulation with high glucose $(17 \mathrm{mM})$ with a microplate reader at $37^{\circ} \mathrm{C}$. CT; control V5

vector. B. Quantification of the area under a curve in $(C)(n=3$; experimental replicates). C. Representative cytoplasmic $\mathrm{Ca}^{2+}$ recording of INS-1 cells transfected with V5-BNLN or FLAG-BNLN after stimulation with high glucose $(17 \mathrm{mM})$. The fluorescent signal was measured in the emission/excitation spectrum of Fluo-8 with FLIPR Tetra System at $37^{\circ} \mathrm{C}$. D. Quantification of the area under a curve in $(E)(n=6-7$; experimental replicates). E. Representative cytoplasmic $\mathrm{Ca}^{2+}$ recording of INS-1 cells overexpressed with FLAG-GFPBNLN before ( $2.5 \mathrm{mM}$ glucose) and after stimulation with high glucose $(17 \mathrm{mM})$ in the absence or presence of $\mathrm{KCl}(40 \mathrm{mM})$. The fluorescent signal was measured in the emission/excitation spectrum of Fluo-8 with a microplate reader at $37^{\circ} \mathrm{C}$. F. Quantification of the area under a curve in $(E)(n=8-9$, experimental replicates). G. ATF6LD-cluc secretion measured in INS-1 cells with BNLN overexpression in the presence of low glucose $(2.5 \mathrm{mM})$ or high glucose $(16.7 \mathrm{mM})$ for $1-2 \mathrm{hr}$ at $37^{\circ} \mathrm{C}$. Data were normalized to Gluc secretion ( $n=13-15$; experimental replicates). H. Representative western blots of BNLN and SERCA3 in HEK293T cells transfected with the indicated constructs for $24 \mathrm{hrs}$. I. PLA assay for SERCA3 interaction with BNLN in INS-1 cells cultured in low glucose $(2.5 \mathrm{mM})$ or challenged with high glucose $(17 \mathrm{mM})$. Scale bar: $10 \mu \mathrm{m}$. -Ab: no antibody control. A model of PLA assay is shown on top of the panel. V5-SERCA3 and FLAG-GFPBNLN were recognized by primary antibodies and secondary antibodies coupled with connector oligos. Data are shown as means \pm SEM. * indicates statistical significance compared to the CT vector group in (B\&D), and compared to treatment of low glucose in cells with same construct in (G); \# indicates statistical significance compared to the CT group in cells treated with the same concentrations of glucose in $(G)$. Statistical significance was determined by student's t-test in (B\&F), and ANOVA followed by Tukey's multiple comparisons test in (D\&G), $p<0.05$. AU: arbitrary unit. 
i73 Figure 5. BNLN regulates glucose-stimulated insulin secretion. A. Glucose stimulated insulin secretion i74 in INS-1 cells overexpressed with FLAG-GFP-BNLN in static incubation in media containing low glucose i75 (2.5 mM) or high glucose (17 mM) for $1 \mathrm{hr}$ each ( $\mathrm{n}=3-4$, experimental replicates). The data were normalized to insulin content. Representative western blots of BNLN expression is shown on the top of pannel. B. GSIS in INS-1 cells overexpressed with the indicated constructs for 48hrs in static incubation in media containing low glucose $(2.5 \mathrm{mM})$ or high glucose $(17 \mathrm{mM})$ for $1 \mathrm{hr}$ each $(\mathrm{n}=3-4$, experimental replicates). The data were normalized to insulin content. C. GSIS measured in isolated islets from mice fed a RD $(n=5-10$, biological replicates; 12 weeks) followed transduction of adeno-GFP (CT) or adeno-BNLN constructs (48 $\mathrm{hrs})$, in static incubation in media containing low glucose $(2.5 \mathrm{mM})$ or high glucose $(17 \mathrm{mM})$ for $1 \mathrm{hr}$ each.

The data were normalized to insulin content. D. Levels of mRNAs encoding for BNLN in islets isolated from mice fed with a RD or a HFD (12 weeks). The data were normalized to Hprt expression in the islets. n=34, biological replicates. E. GSIS measured in isolated islets from mice fed a HFD ( $n=3-4$, biological replicates; 12 weeks on HFD) followed transduction of adeno-GFP (CT) or adeno-BNLN constructs (48hrs), in static incubation in media containing low glucose $(2.5 \mathrm{mM})$ or high glucose $(17 \mathrm{mM})$ for $1 \mathrm{hr}$ each. The data were normalized to insulin content. F-H. Representative GSIS measured in islets from human donors followed transduction of adeno-GFP (CT) or adeno-BNLN constructs (48hrs), in static incubation in media containing low glucose $(2.5 \mathrm{mM})$ or high glucose $(17 \mathrm{mM})$ for $1 \mathrm{hr}$ each. The data were normalized to insulin content. In (F), GSIS was performed on a male non-diabetic human donor of 69 years of age, 27.2 BMI, in (G), GSIS was performed on a male non-diabetic human donor of 18 years of age, 19 BMI. I. Insulin content measured in islets from human donors followed transduction of adeno-GFP (CT) or adeno-BNLN constructs (48hrs). Data are represented as a difference in intracellular insulin content after performing GSIS between CT and BNLN human islets. Data are shown as means \pm SEM. *indicates statistical significance compared to treatment of low glucose in cells/islets with same type of construct in $(A, C, E \& H)$, to the CT vector group in (B); \# indicates statistical significance compared to the CT group in cells/islets i97 incubated with the same concentrations of glucose. Statistical significance was determined by student's ttest in (D\&H), and ANOVA followed by Tukey's multiple comparisons test in (A\&B\&C\&E), $p<0.05 . A U$ : arbitrary unit. 
'01 Supplementary Material:

'02 Supplemental Figure 1. Expression of TUNAR in various human tissues. Violin plot of TUNAR RNA '03 expression across various human tissues. Expression values are measured by log10(TPM+1), data from '04 GTEX database.

'05

'06 Supplemental Figure 2. Characterization of BNLN. A. Ribosomal (Ribo-seq) profiling track from GWIPS'07 viz genome browser indicating translation of sORF at the begining of the last exon. B. TMHMM algorithms '08 predict a transmembrane domain at the C-terminus of BNLN.

'09

'10 Supplemental Figure 3. Conservation of BNLN across vertebrates species. 88 species out of 100 '11 vertebrate species (UCSC multiz100way) contain homologous sORFs. Multi-species alignment of in silico '12 translated putative BNLN protein sequence. 


\section{Figure 1}

A

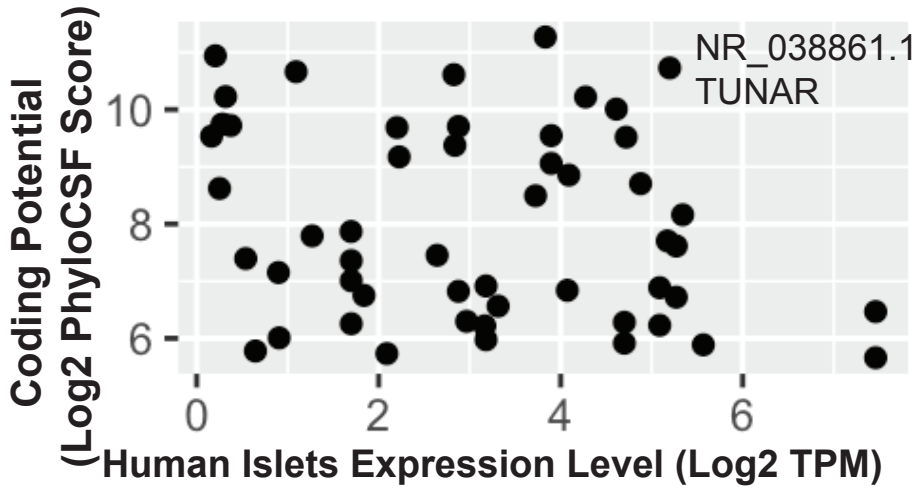

TUNAR $49.2 \mathrm{~kb}$

C

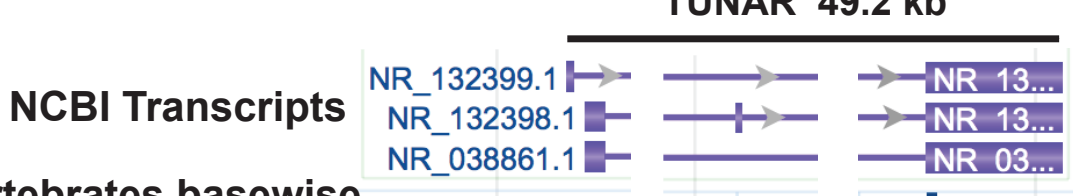

100 Vertebrates basewise NR_038861.1 - - NR 03..

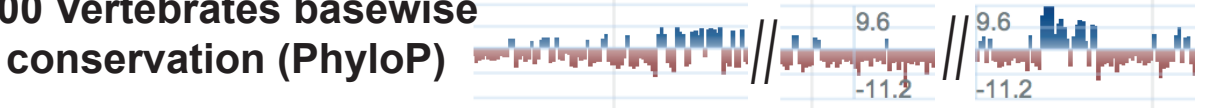

NCBI Aggregate RNA-seq Coverage (log 2 scaled)

\begin{tabular}{|c|c|c|}
\hline Gene & $\begin{array}{l}\text { PhyloCSF } \\
\text { Score }\end{array}$ & $\begin{array}{l}\text { RNAcode } \\
\text { Score }\end{array}$ \\
\hline SPAAR(SPAR) & 857 & 43 \\
\hline NBDY & 386 & 32 \\
\hline STRIT1(DWORF) & 406 & 23 \\
\hline MYMX & 1344 & 29 \\
\hline C4orf3(ALN) & 738 & 24 \\
\hline SMIM6(ELN) & 58 & 18 \\
\hline MRLN & 265 & 9 \\
\hline MTLN & 1192 & 19 \\
\hline APELA & 262 & 18 \\
\hline C12orf75 & 678 & 17 \\
\hline CYREN & 605 & 17 \\
\hline HOXB-AS3 & -188 & 28 \\
\hline TUNAR* & 1696 & 23 \\
\hline
\end{tabular}

D

Predicted Human Seq

V1st ATG Y2nd ATG $\quad$ 3rd ATG

Consensus / $100 \%$

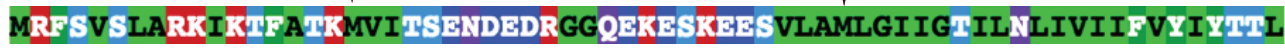

Consensus I $90 \%$

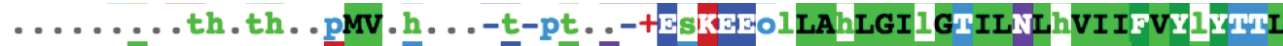

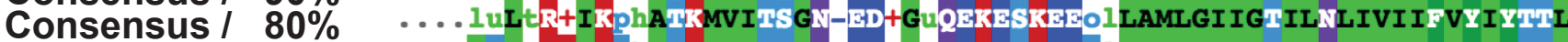

Consensus I $70 \%$

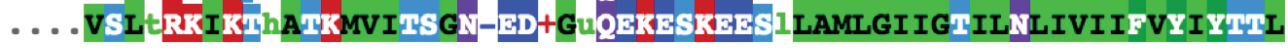


bioRxiv preprint doi: https://doi.org/10.1101/2020.05.12.091728; this version posted July 26, 2020. The copyright holder for this preprint (which - 1 Ure 2 was not certified by peer review) is the author/funder. All rights reserved. No reuse allowed without permission.

A

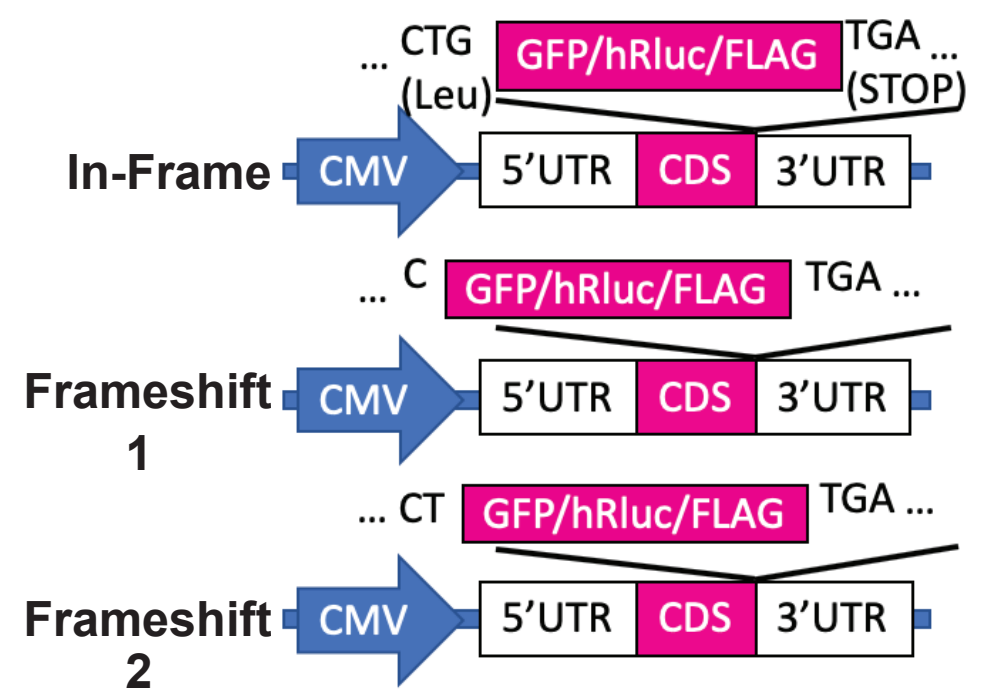

In-Frame-GFP Frameshift-1 Frameshift-2

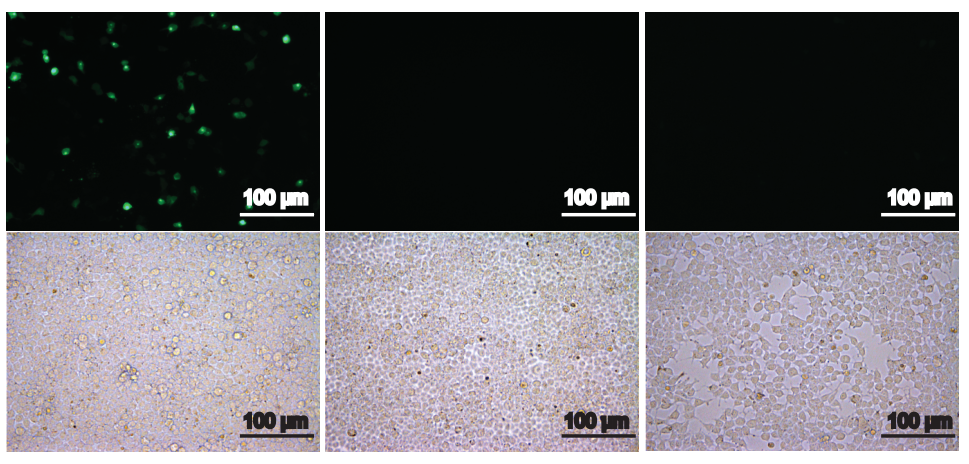

C

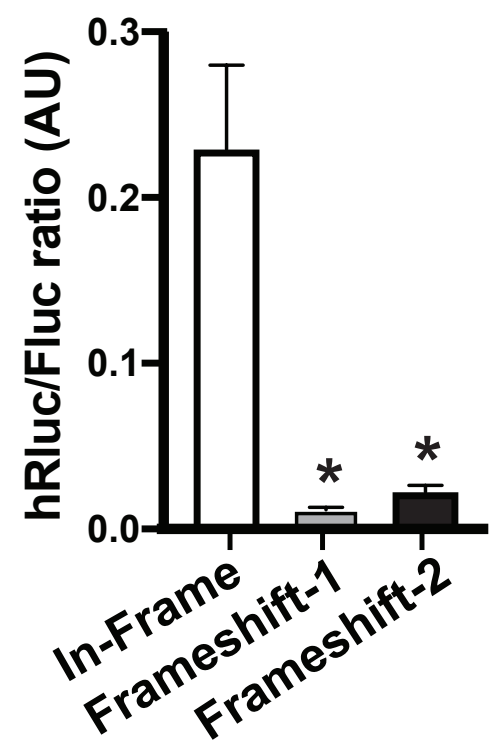

D

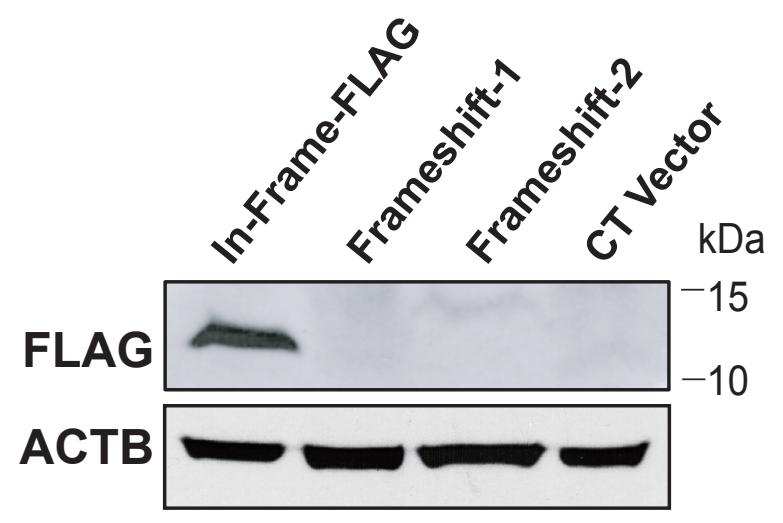

E

$\mathbf{F}$
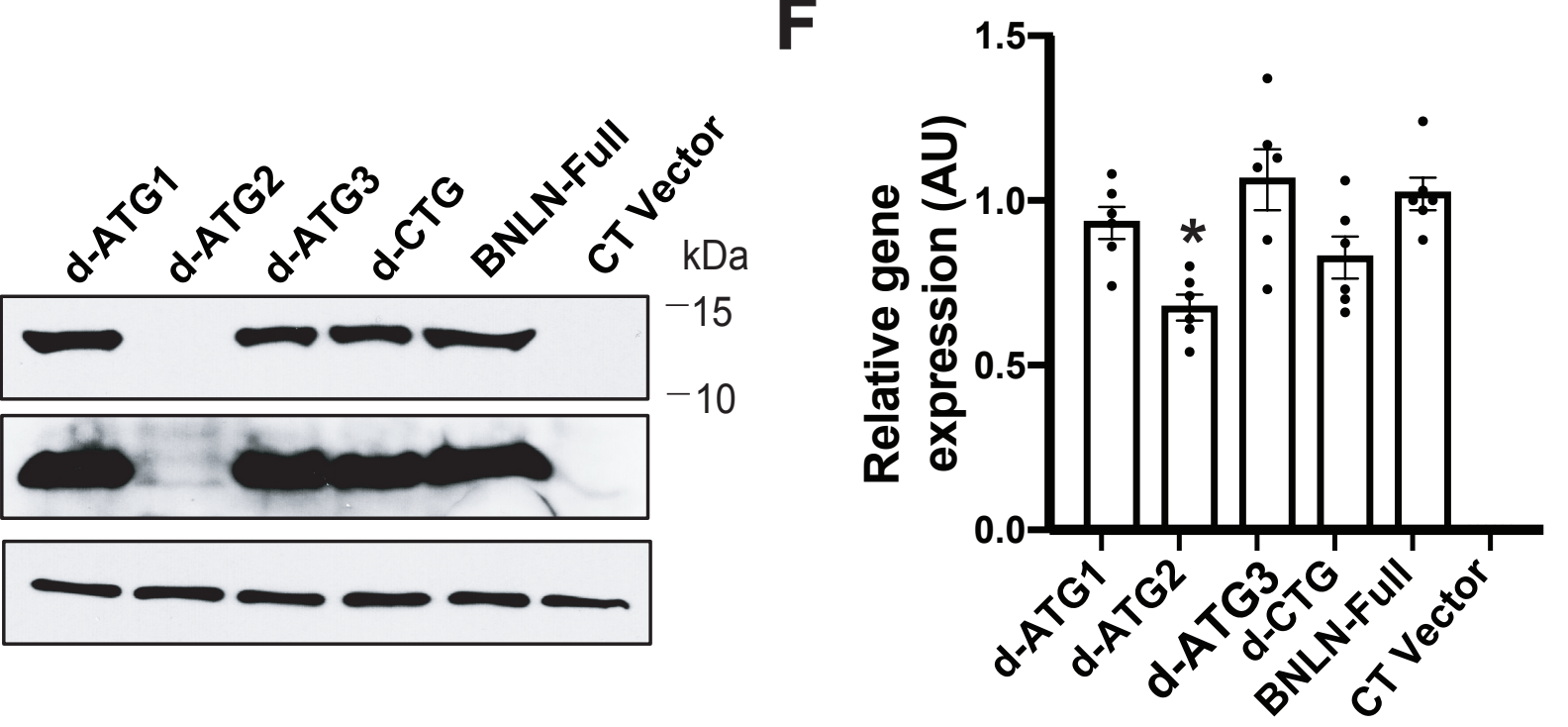
$\mathrm{kDa}$
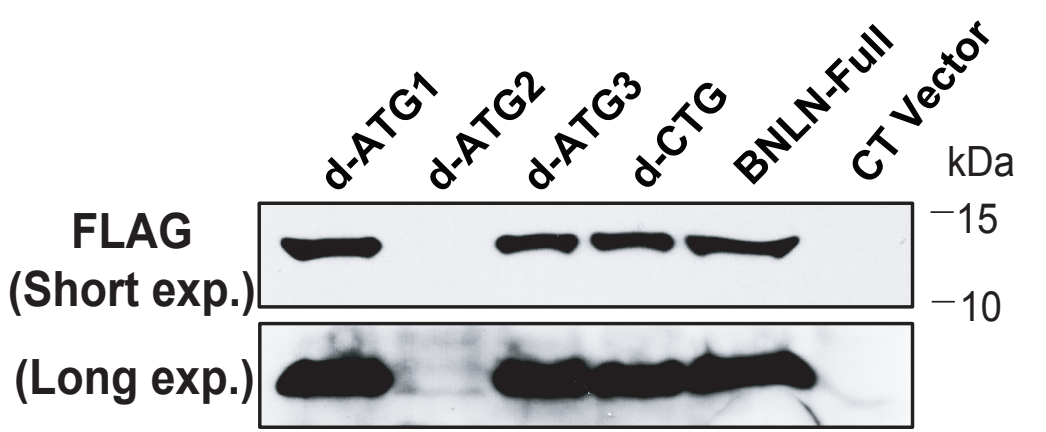
A
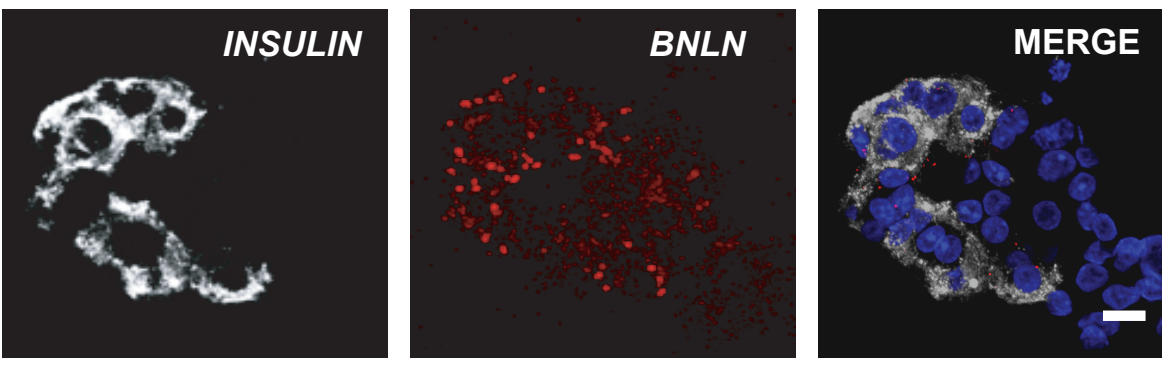

B

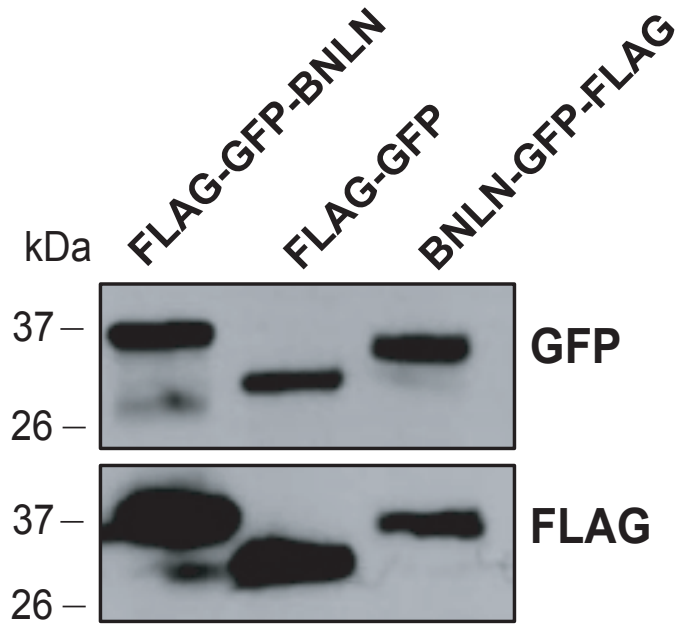

D

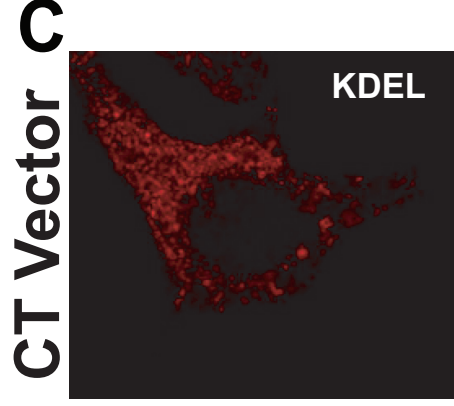

KDEL
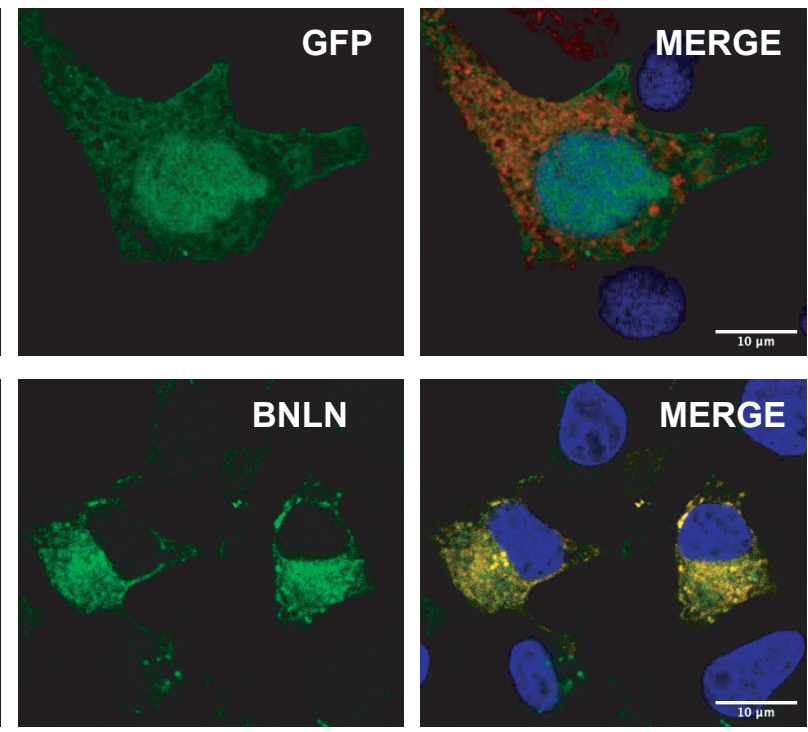

E

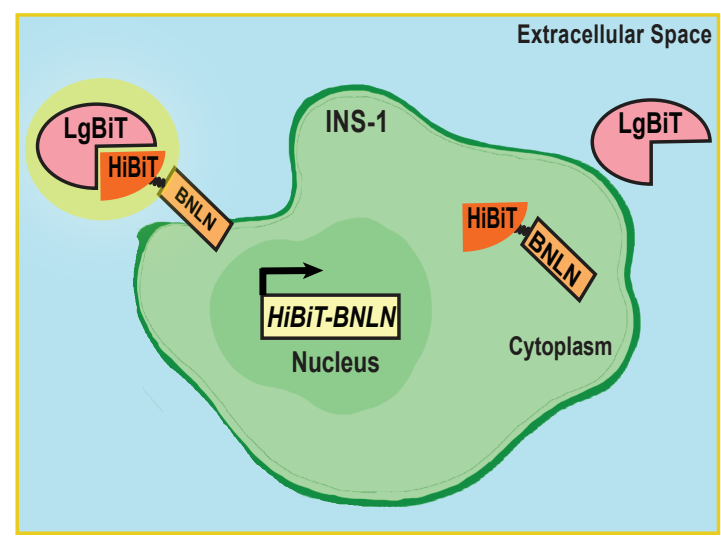

F

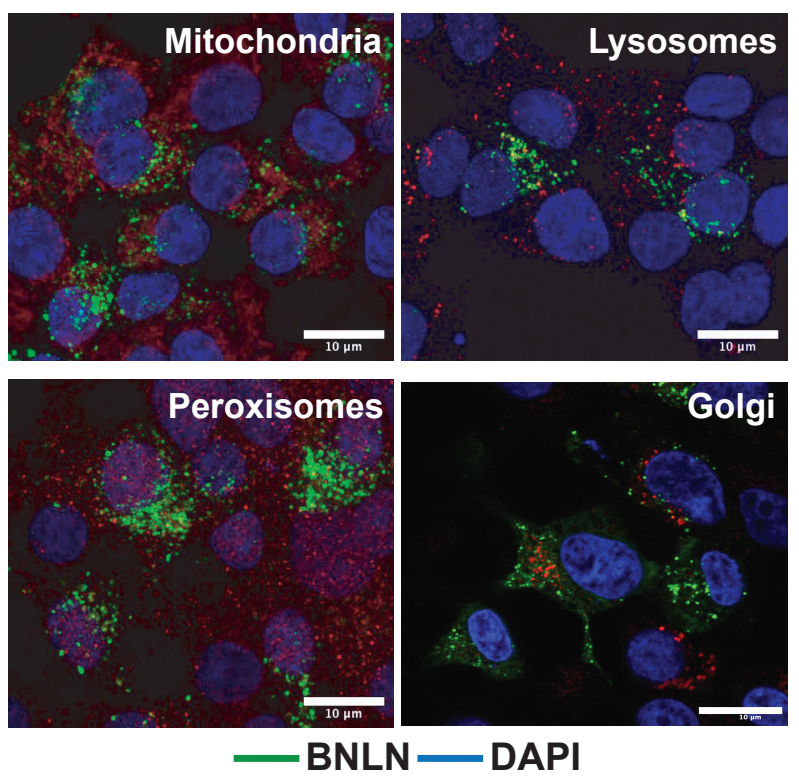

- Intracellular signal

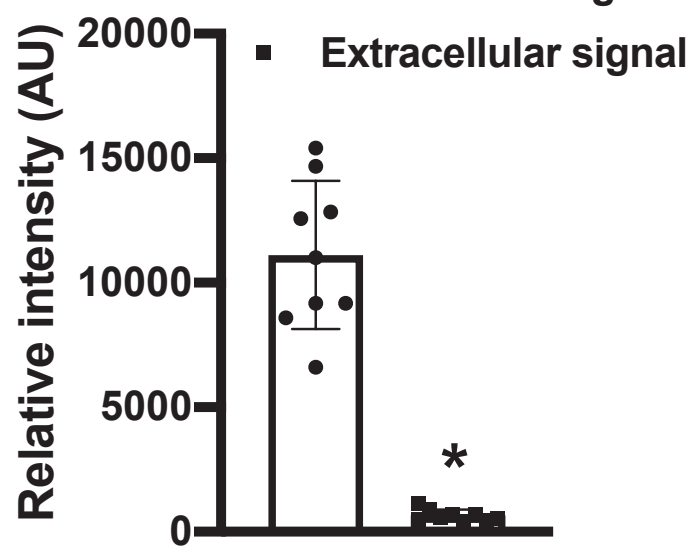


bioRxiypreprint doi: https://doi.org/10.1101/2020.05.12.091728; this version posted July 26, 2020. The copyright holder for this preprint (which

E 10 was not certified by peer review) is the author/funder. All rights reserved. No reuse allowed without permission.

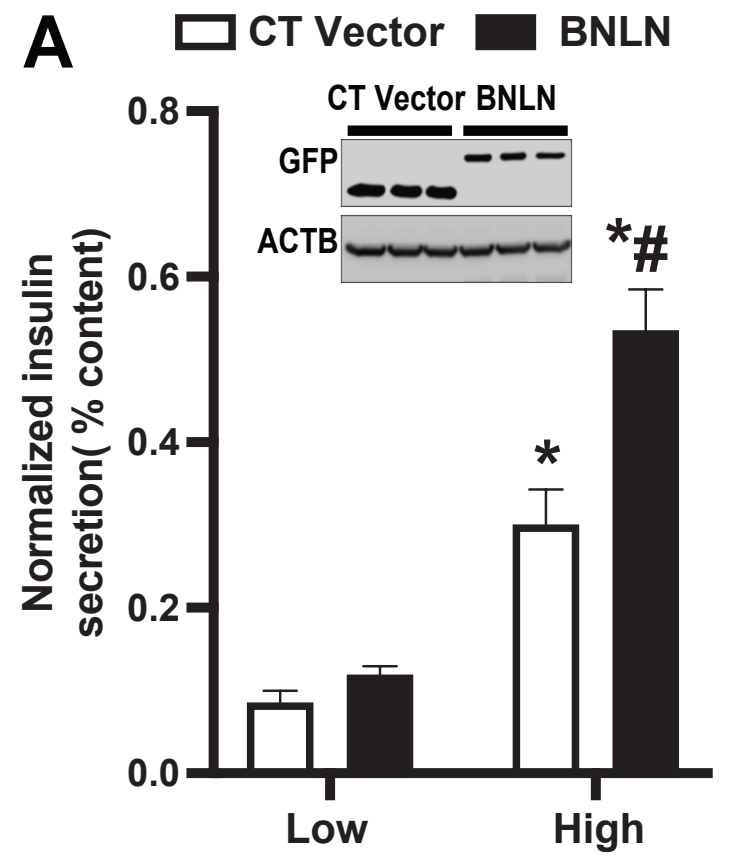

D
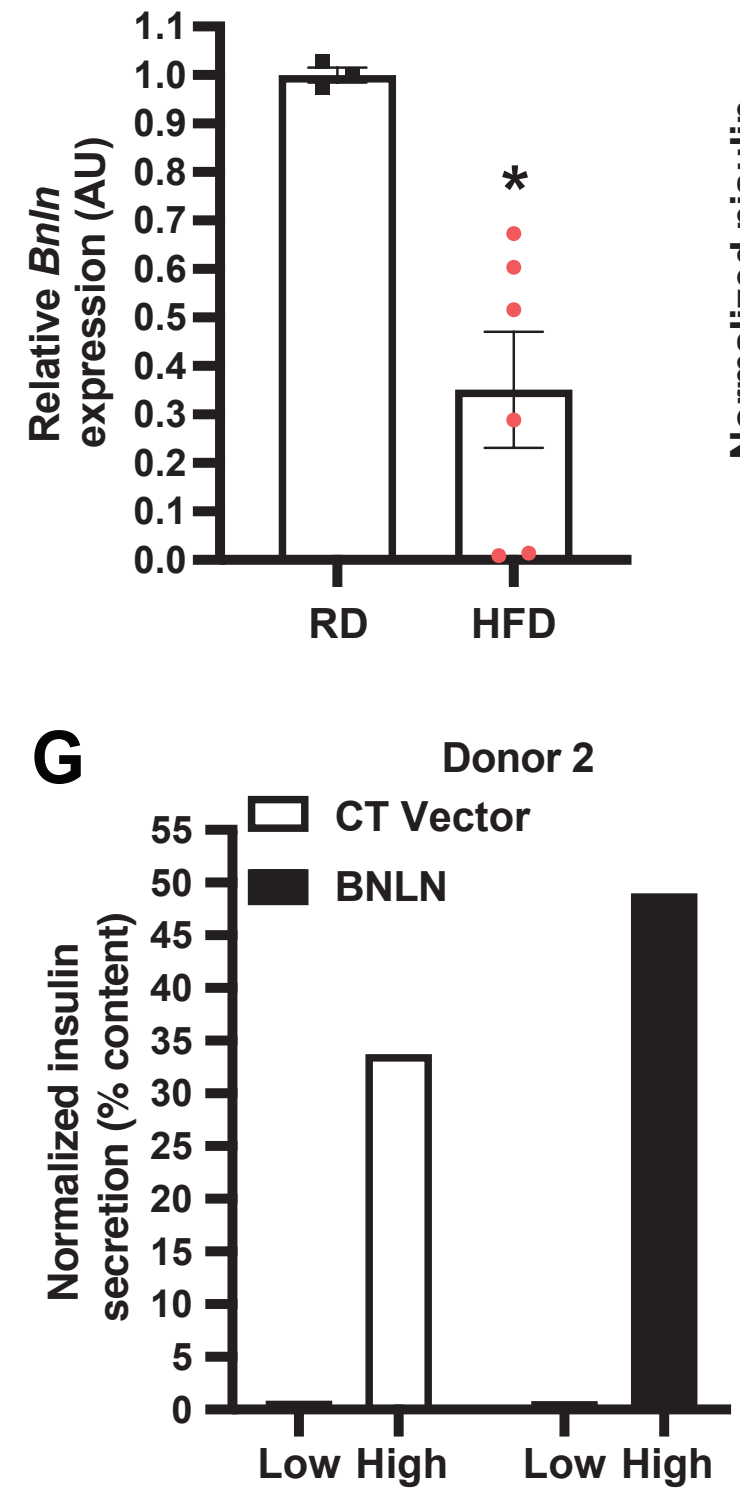

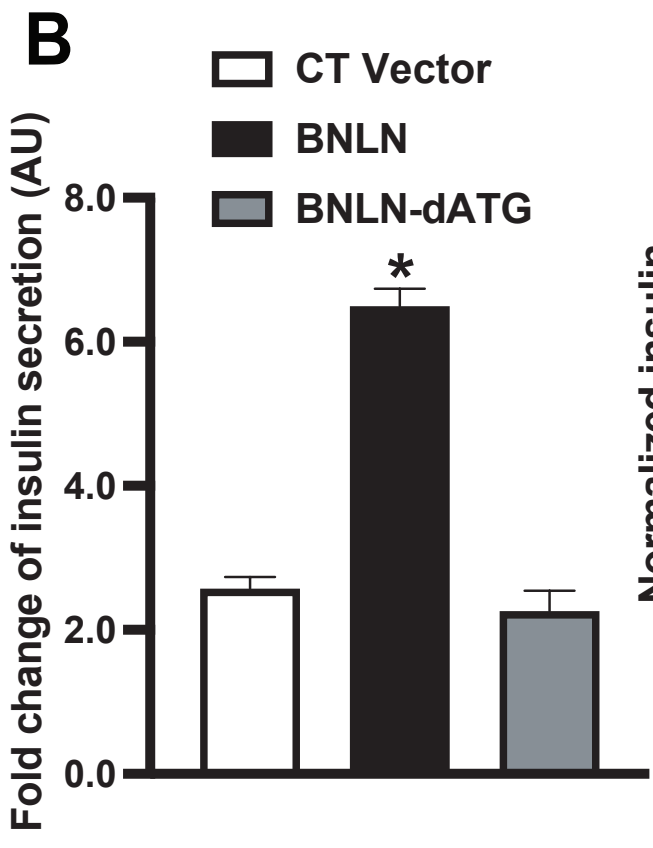

C
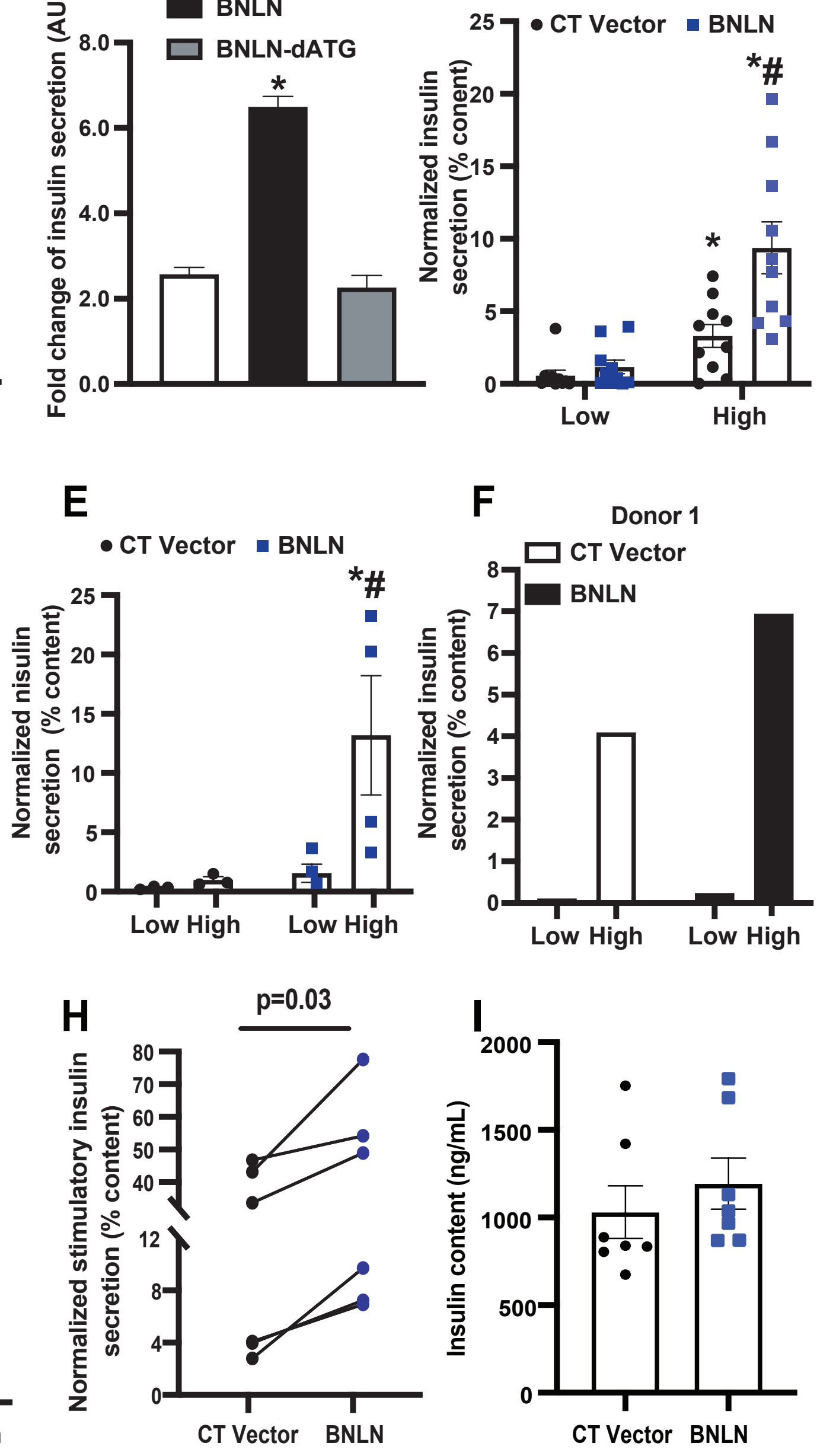